\title{
Saline: An API for Thermophysical Properties
}

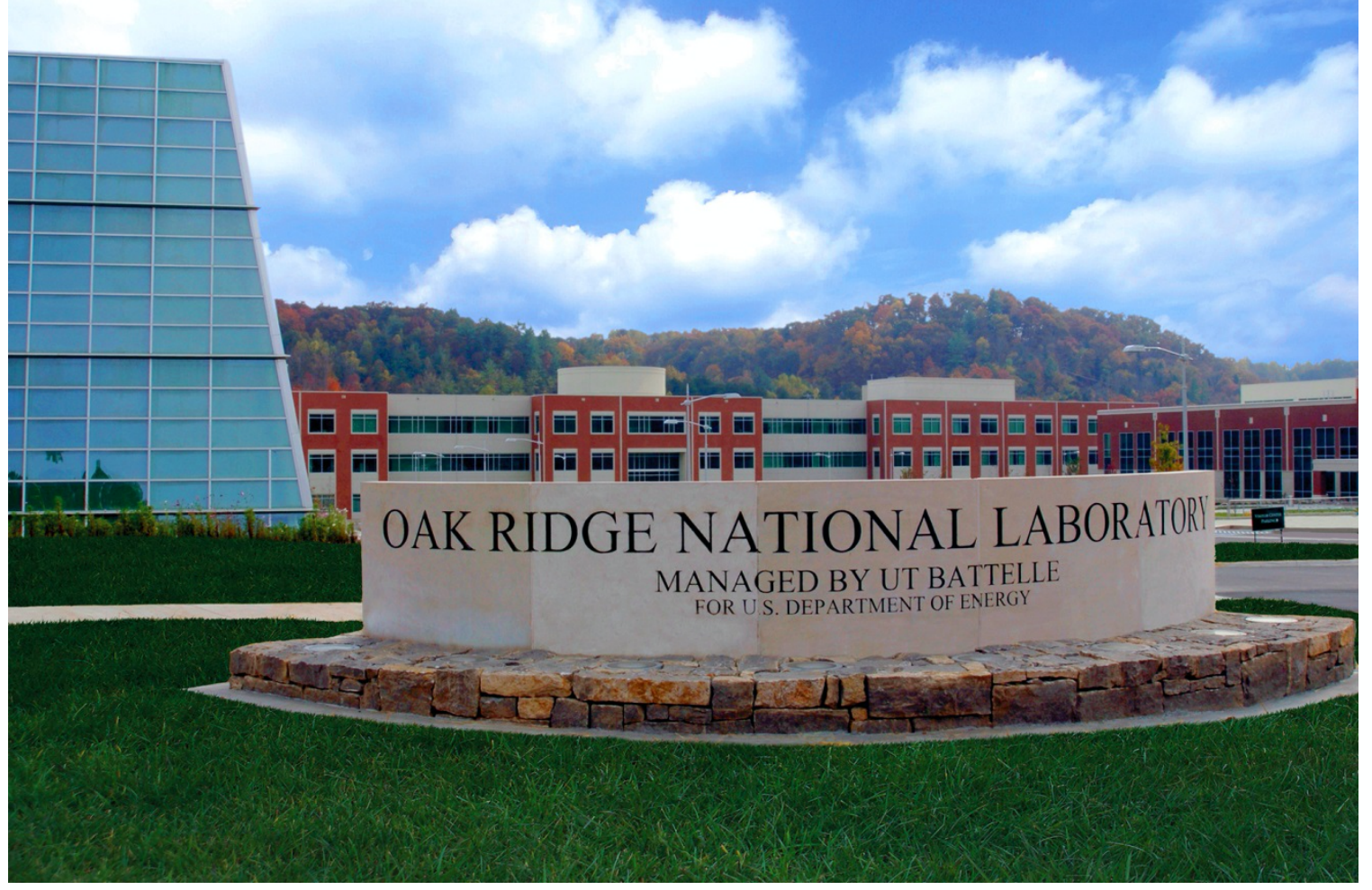
S. Henderson
C. Agca
J. Mcmurray
R. Lefebvre

September 292021

Approved for public release.

Distribution is unlimited. 


\title{
DOCUMENT AVAILABILITY
}

Reports produced after January 1, 1996, are generally available free via US Department of Energy (DOE) SciTech Connect.

Website www.osti.gov

Reports produced before January 1, 1996, may be purchased by members of the public from the following source:

\author{
National Technical Information Service \\ 5285 Port Royal Road \\ Springfield, VA 22161 \\ Telephone 703-605-6000 (1-800-553-6847) \\ TDD 703-487-4639 \\ Fax 703-605-6900 \\ E-mail info@ntis.gov \\ Website http://classic.ntis.gov/
}

Reports are available to DOE employees, DOE contractors, Energy Technology Data Exchange representatives, and International Nuclear Information System representatives from the following source:

Office of Scientific and Technical Information

PO Box 62

Oak Ridge, TN 37831

Telephone 865-576-8401

Fax 865-576-5728

E-mail reports@osti.gov

Website https://www.osti.gov/

This report was prepared as an account of work sponsored by an agency of the United States Government. Neither the United States Government nor any agency thereof, nor any of their employees, makes any warranty, express or implied, or assumes any legal liability or responsibility for the accuracy, completeness, or usefulness of any information, apparatus, product, or process disclosed, or represents that its use would not infringe privately owned rights. Reference herein to any specific commercial product, process, or service by trade name, trademark, manufacturer, or otherwise, does not necessarily constitute or imply its endorsement, recommendation, or favoring by the United States Government or any agency thereof. The views and opinions of authors expressed herein do not necessarily state or reflect those of the United States Government or any agency thereof. 
Nuclear Energy and Fuel Cycle Division

\title{
SALINE: AN API FOR THERMOPHYSICAL PROPERTIES
}

\author{
S. Henderson \\ C. Agca \\ J. Mcmurray \\ R. Lefebvre
}

September 2021

Prepared by

\author{
OAK RIDGE NATIONAL LABORATORY \\ Oak Ridge, TN 37831-6283 \\ managed by \\ UT-BATTELLE LLC \\ for the \\ US DEPARTMENT OF ENERGY \\ under contract DE-AC05-00OR22725
}





\section{CONTENTS}

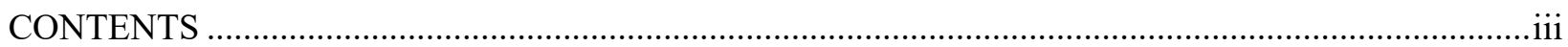

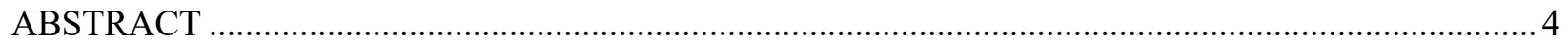

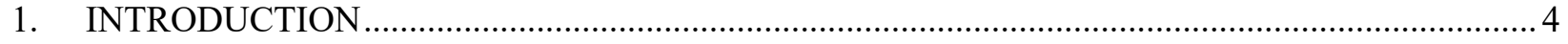

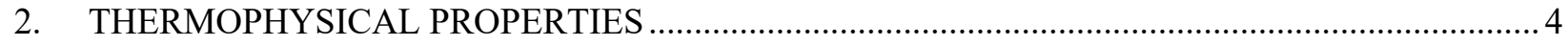

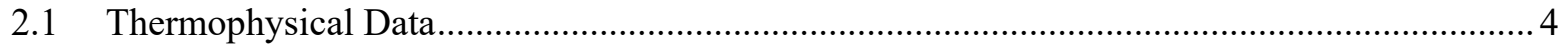

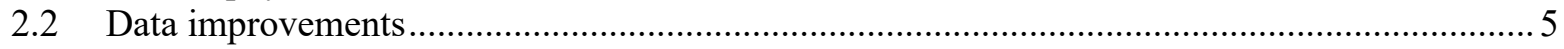

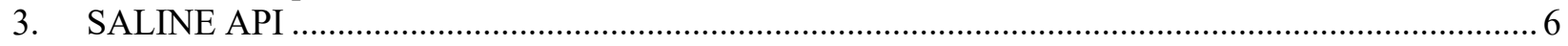

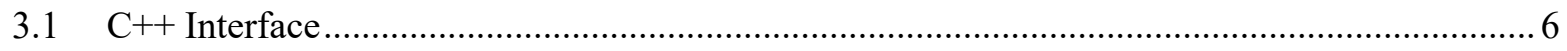

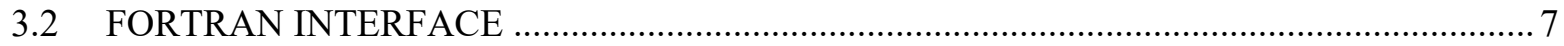

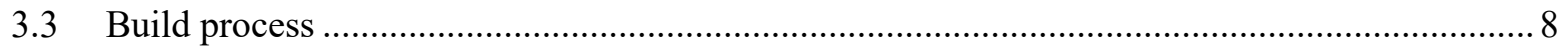

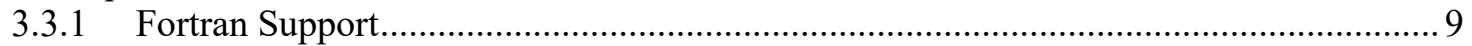

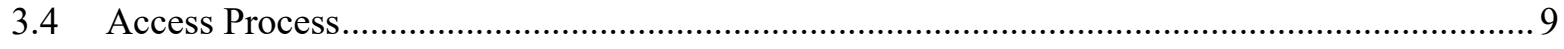

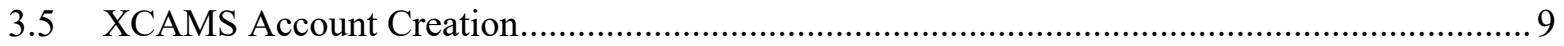

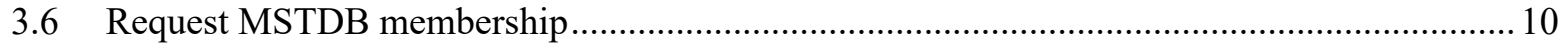

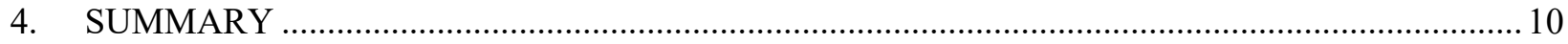

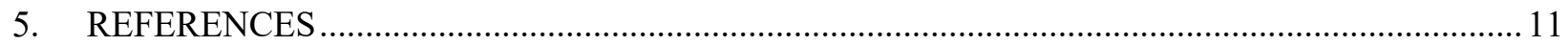

APPENDIX A. MOLTEN SALT THERMOPHYISCAL DATA …….............................................

APPENDIX B. MOLTEN SALT THERMOPHYISCAL DATA ….................................................... B-3 


\begin{abstract}
An initial interface for thermophysical data was generalized for broader incorporation into modeling and simulation codes used for molten salt reactor analysis. A quality assurance review of the thermophysical properties data was conducted; errors were fixed, and improvements were made with consistency and accuracy to references. The Saline code application programming interface was created to facilitate access and integration of thermophysical properties. This report describes the thermophysical data, the quality assurance improvements, and the Saline interface.
\end{abstract}

\title{
1. INTRODUCTION
}

Saline's predecessor, an initial interface for thermophysical data [1], was generalized into a C++ application programming interface (API) with Fortran interface bindings for broader incorporation into modeling and simulation codes. In addition, the thermophysical data used by the API was improved.

\section{THERMOPHYSICAL PROPERTIES}

Thermophysical properties of molten salts are important for the reactor design, operation, and safety [2]. Obtained from the thermal analyses or CALPHAD (Computer Coupling of Phase Diagrams and Thermochemistry) method, the melting and the boiling temperatures of the salts provide liquid-phase stability range. Density, viscosity, thermal conductivity, and heat capacity are important transport properties in determining parameters such as flow regimes, heat transfer, and volumetric flow rates [3]. For engineering design, thermophysical data and associated uncertainty values are required. Development of a thermophysical database is a utilitarian way to organize this information for use by end users with, for example, thermal hydraulics or multiphysics reactors simulation software.

\subsection{THERMOPHYSICAL DATA}

Thermophysical properties of molten salts have been studied by various authors, and the measured data have been published [4]-[10]. The data and the experimental measurements or theoretical estimation methods used to generate them were compiled in several publications [11]-[17]. Janz et al. prepared several reports on the existing thermophysical property data over the years [18]-[23]. Serrano-Lopez et al., Tasidou et al., and Magnusson et al. have compiled the most recent data [11],[16],[24]. These data sets and reports have been useful for finding the original data for further details such as measurement technique and error margins.

MSTDB-TP (Molten Salt Thermal Database-Thermophysical) [25],[26] was developed as an extension of the work performed by Jerden [15]. The report documented the first version of MSTDB-TP, an Excel spreadsheet. The current version of MTSDB-TP aims to improve the data with the source reference publication. It contains 62 entries. Of them, 27 are pure compounds, 8 are pseudobinary, 10 are pseudoternary and 5 are pseudoquaternary molten salt mixtures at specific compositions. The pure compounds and specific composition mixtures comprise fluorides and chlorides that are of interest to the molten salt reactor community. The following properties of the molten salts are provided.

- Melting and boiling temperatures

- Density

- Viscosity

- Thermal conductivity

- Heat capacity 
Estimation of measurement errors are provided along with the original reference for each property. The melting temperature of almost all (61/62) of the compounds are included. Boiling temperatures are scarce in the literature for entries with two and more components in MSTDB-TP; currently, the database has 32 boiling point records out of 62 entries. Additional vapor pressure measurements and phase equilibrium calculations can help determine these boiling points. Most entries have density (54/62) and viscosity (48/62) records in the database. Heat capacity of all pure compounds and some of the molten salt mixtures are included (43/62). The scarcest property in the database is thermal conductivity (25/62). The scarcity stems from the inconsistency of the measurement results from different studies and insufficient modeling, for which a variable gap method was developed to provide data [27]-[29].

\subsection{DATA IMPROVEMENTS}

The Jerden report [15] was accompanied with an Excel spreadsheet. Quality assurance was performed on this first version of the spreadsheet. The data were compared with the original reference, and inconsistencies in the values and units were found and fixed. To ensure that the data were consistent, each property was compared with different literature values where possible. When the original data conflicted with the literature, more consistent values with lower uncertainties were selected.

Overall, the thermophysical data of molten salts are sparse, and the measurement results have different uncertainties. The laboratories that conducted the measurements have different standards, operational procedures, measurement techniques, sample purities, and sample characterization techniques that affect the thermophysical data results. Furthermore, simple mathematical expressions are needed that can describe the thermophysical properties of molten salts with respect to temperature and composition.

This type of mathematical modeling has been implemented for the density of $\mathrm{NaCl}-\mathrm{KCl}-\mathrm{UCl}_{3}$ and $\mathrm{NaCl}-$ $\mathrm{KCl}-\mathrm{YCl}_{3}$ molten salt mixtures successfully in comparison with the experimental data [29]. The RedlichKister expansion [30] has been used to account for the nonideal behavior in density. The same procedure can be applied for viscosity and thermal conductivity. Ideal behavior equations for density, viscosity, and thermal conductivity of binary liquids are given in Eqs. (1-3).

$$
\begin{gathered}
\rho_{i d}=\frac{x_{A} M W_{A}+x_{B} M W_{B}}{\frac{x_{A} M W_{A}}{\rho_{A}}+\frac{x_{B} M W_{B}}{\rho_{B}}} \\
\log \mu_{i d}=x_{A} \log \mu_{A}+x_{B} \log \mu_{B} \\
\lambda_{i d}=x_{A} \lambda_{A}+x_{B} \lambda_{B}
\end{gathered}
$$

The ideal density is the ratio of molar mass to molar volume as shown in Eq. (1), where $x_{i}$ is the mole fraction of $i, M W_{i}$ is the molecular weight of $i$, and $\rho_{i}$ is the density of pure $i$. Ideal viscosity has a $\log$ dependence with respect to composition as demonstrated by Arrhenius [32] in Eq. (2), where $\mu_{i}$ is the viscosity of $i$. Thermal conductivity ideal behavior was adopted from Dul'nev and Zarichnyak [32]. Thermal conductivity of a liquid depends on the heat capacity at constant volume $\left(C_{v}\right)$, heat capacity at constant pressure $\left(C_{p}\right)$, and velocity of sound of the molten salts [34],[35]. Data are available for various molten salts in the literature [27],[36]. Therefore, Eq. (3) must be revised with accurate heat capacity and velocity data. Heat capacity is modeled as a part of MSTDB-TC (Molten Salt Thermal DatabaseThermochemical) and is calculated using the CALPHAD method [37].

Nonideal behavior of density, viscosity, and thermal conductivity are described by the Redlich-Kister expansion [31]. Equation (4) is added as a nonideal behavior term $\left(\rho_{\text {nid }}, \mu_{\text {nid }}, \lambda_{\text {nid }}\right)$ in Eqs. $(1-3)$.

$$
\rho_{\text {nid }}, \mu_{\text {nid }}, \lambda_{\text {nid }}=x_{A} x_{B} \sum_{j=1}^{n} L_{j}\left(x_{A}-x_{B}\right)^{j-1}
$$


$L_{j}$ is the interaction term that accounts for the nonideality. As shown in Eq. (5), it has a linear dependence with respect to temperature.

$$
L_{j}=A_{j}+B_{j} T
$$

The parameters $A_{j}$ and $B_{j}$ are parameter constants, which are specific to molten salt mixtures. The interaction parameters that are modeled from binary molten salts can be used to extrapolate to ternary and higher order molten phases using the Muggianu interpolation scheme [30][38][39].

The Redlich-Kister modeling approach outlined here will provide broader composition coverage and temperature range for coolant, carrier, or fueled molten salts. However, it requires experimental results or theoretical estimations on binary molten salt density, viscosity, and thermal conductivity as inputs and validating, confirmatory data. Thermophysical measurements need to be conducted on planned binary molten salt systems described in the roadmap by McMurray et al. [25]. If there are no thermophysical data on binary salts, one can estimate the properties using the ideal behavior equations from pure molten salts shown in Eqs. (1)-(3), which are outlined in MSTDB-TP.

\section{SALINE API}

Saline is an API for the quality-assured collection of molten salt thermophysical property data described in Section 2. It provides a collection of classes and methods to facilitate the integration of molten salt density, viscosity, thermal conductivity, and heat capacity data models into a broad collection of client applications. The interface is written in $\mathrm{C}++11$ with Fortran bindings. Python language bindings are in draft form. The configuration and compilation of Saline is managed using CMake for support across operating systems.

\subsection{C++ INTERFACE}

Saline is an object-oriented application predominately written using $\mathrm{C}++11$. The main class of interest is the Thermophysical_Property class. This class provides for setting a particular composition via the setCompostion method and looking up data provided for that composition via data accessors. A data accessor is provided for looking up each thermophysical property using either temperature in Kelvin or enthalpy in Joule per mole. Users are encouraged to use the temperature interface if possible. Currently, Saline does not check to ensure that the requested temperature/enthalpy is within the range of the data model. In subsequent versions of Saline, a warning or exception may be raised when an out-of-range request is made.

The data models employed by Thermophysical_Properties are implemented in a Data_Store class. This class is intended to be extensible to provide alternative data implementations to client codes without requiring substantial changes within the client. Although additional data sets are under development, only use of the Default_Data_Store is currently recommended. This class makes the data discussed in Section 2 available for use.

An additional data set that may be used with caution is the R_Kister_Data_Store. This Data_Store uses the binary data provided by the Default_Data_Store and applies a Redlich-Kister polynomial to support arbitrary salt compositions. This provides for a very robust interface, but currently, only the density models of specific salts are implemented. All other properties are still obtainable, but the provided data are obtained using an ideal mixing assumption with unquantified uncertainty. 


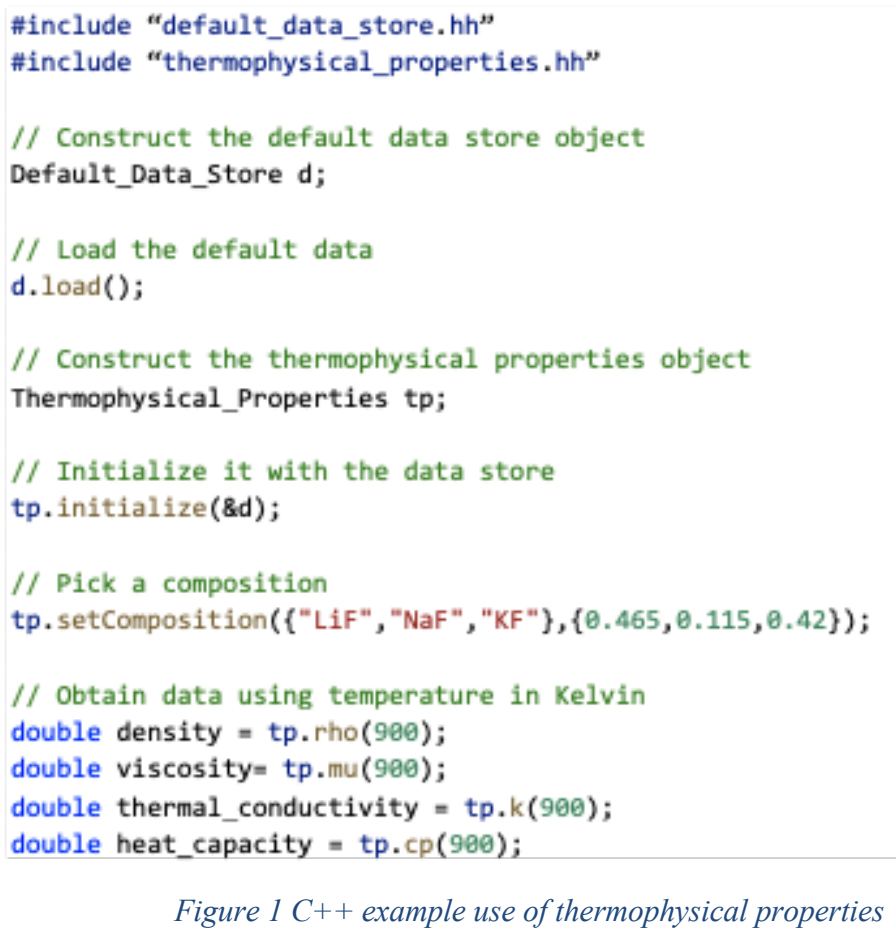

Figure 1 C++ example use of thermophysical properties

\subsection{FORTRAN INTERFACE}

Saline also provides a Fortran interface for use where required. This interface is a wrapper class that uses the ISO_C_Binding module to pass Fortran arguments back and forth. Aside from the syntax of the programming language, only a select few methods have a noticeable difference, which are listed as follows.

1. The data accessors argument for pressure is a required argument with no effect on output value.

2. setComposition takes a single character array for salt names, an array of doubles for mole percentages, and a count of the number of constituents. Salt names should be delimited with a hyphen (-). 


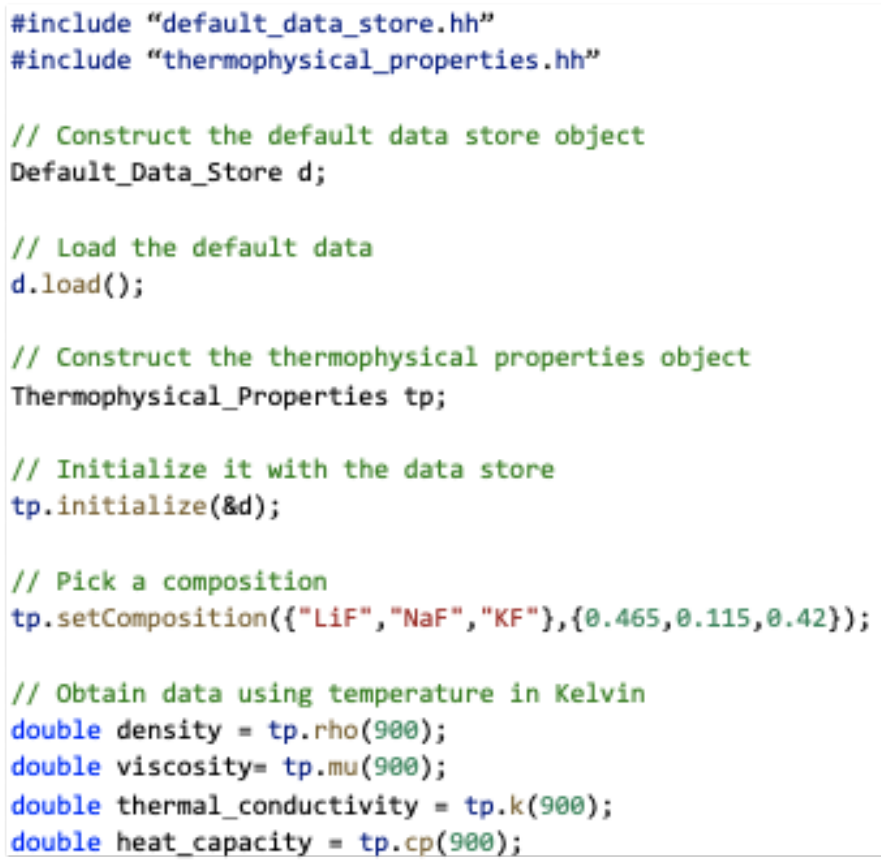

Figure 2 Fortran example use of the thermophysical properties

\subsection{BUILD PROCESS}

Saline was developed to utilize the CMake-based TriBITS build integration and test system. This system should enable Saline to be added to other TriBITS or CMake projects with minimal effort. The TriBITS documentation provides additional details for users in this circumstance.

The process outlined as follows will build Saline into a library, libSaline.so, which may be linked for use in other codes.

- Meet the prerequisites

- $\mathrm{C}++11$ compliant $\mathrm{C} / \mathrm{C}++$ compiler

○ Cmake 3.1+

○ Git $2.15+$

○ Python 2.6+ (Python3+ preferred) - optional

- Set up a source directory using the following commands

o Clone saline git clone https://code.ornl.gov/neams/saline.git /saline

- Change directory into saline $\mathrm{cd} \sim /$ saline

- Clone TriBITS git clone https://github.com/lefebvre/TriBITS.git TriBITS

- Clone extra repos./TriBITS/tribits/ci_support/clone_extra_repos.py

- Create a build directory mkdir -p /build/saline

$\circ$ Change into the build $\mathrm{cd} \sim / \mathrm{build} / \mathrm{saline}$ 
- Create the recommended configuration script. For this example, the script is called configure.sh and is saved in the $\sim$ build directory.

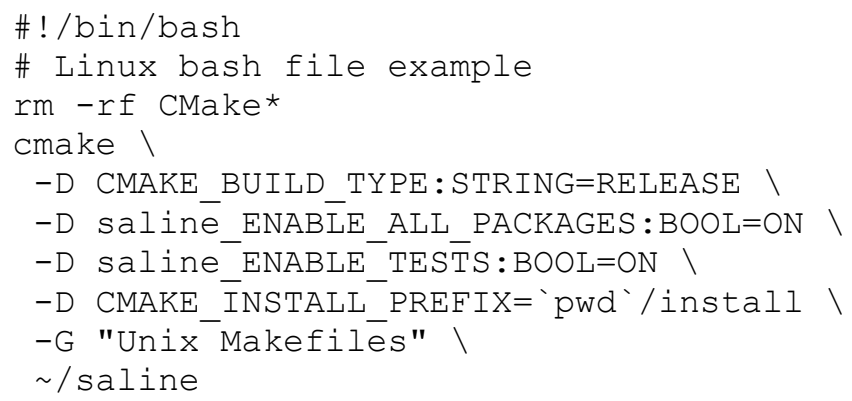

- Execute the following to configure, build, and test from the $\sim /$ build/saline directory.

○ Configure Saline va .. /configure.sh

- Compile Saline via make

- Run the Saline tests via ctest

\subsubsection{Fortran Support}

To enable Fortran support, execute the command, export saline_ENABLE_Fortran=ON, before running the configure script. Compiling the Fortran code requires an F2003-compliant Fortran compiler.

\subsection{ACCESS PROCESS}

The Saline project and associated thermophysical data are hosted on a publicly accessible server at Oak Ridge National Laboratory (ORNL), https://code.ornl.gov/neams/saline/. Access to this server requires an ORNL XCAMS account and a Saline project membership.

\subsection{XCAMS Account Creation}

XCAMS allows external, non-ORNL users to access code.ornl.gov. XCAMS accounts are created as follows:

1. Go to https://xcams.ornl.gov.

2. Select "I need an account."

3. Read and acknowledge the User Agreement.

4. Enter your email address and username, following the guidelines provided on the page.

5. Enter "Personal Information" and "Contact Information" according to the guidelines provided on the page.

6. Create an XCAMS password according to the guidelines provided on the page.

7. On the final step, note the activation sequence box. Wait until each action item turns green and the box heading reads "Transactions Complete." 


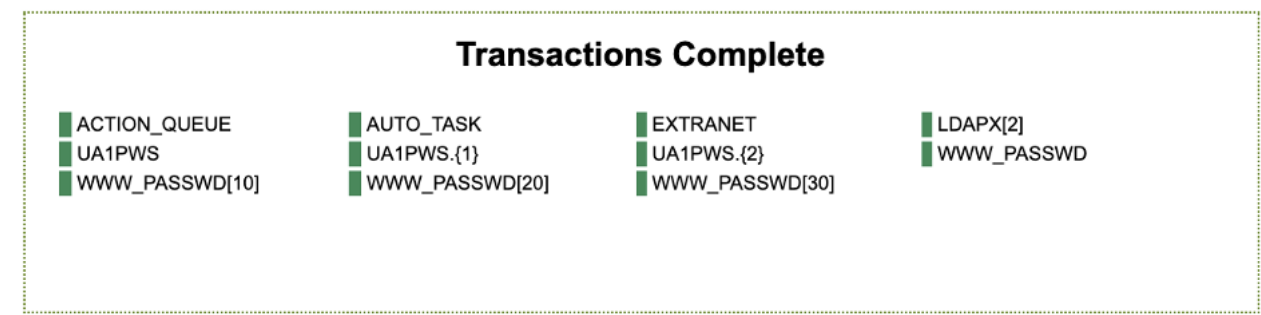

8. Log in to https://code.ornl.gov using your new XCAMS username and password.

\subsection{Request MSTDB membership}

Send an email to Rob Lefebvre, lefebvrera@ornl.gov, with subject "Saline Access Request," and include the following information:

1. Your XCAMS ID

2. A summary of the purpose of your access

You will receive an email from code.ornl.gov indicating your Saline access has been granted and a link to the project page.

\section{SUMMARY}

This milestone report highlights improvements to thermophysical properties data and the creation of the Saline API. The Saline API was created to provide capabilities that can be integrated into $\mathrm{C}++$ and Fortran molten salt reactor modeling and simulation codes. 


\section{REFERENCES}

[1] Salko, Jr., R.; Graham, A. C.; Lefebvre, R. A.; Langley, B. R. Demonstration of MSR salt property database operation with a reactor analysis tool. ORNL/TM-2019/1362. Oak Ridge National Laboratory, 2019. https://doi.org/10.2172/1615804.

[2] Abou-Jaoude, A.; Harper, S.; Giudicelli, G.; Balestra, P.; Schunert, S.; Martin, N.; Lindsay, A.; Tano, M.; Freile, R. "A Workflow Leveraging MOOSE Transient Multiphysics Simulations to Evaluate the Impact of Thermophysical Property Uncertainties on Molten-Salt Reactors." Ann. Nucl. Energy 2021, 163, 108546. https://doi.org/10.1016/j.anucene.2021.108546.

[3] Gurecky, W.; de Wet, D.; Greenwood, M.; Salko, Jr., R.; Pointer, D. Coupling of CTF and TRANSFORM Using the Functional Mockup Interface. ORNL/TM-2020/1872. Oak Ridge National Laboratory, 2020. https://doi.org/10.2172/1763465.

[4] Cantor, S. Density and Viscosity of Several Molten Fluoride Mixtures. ORNL-TM-4308. Oak Ridge National Laboratory, 1973. https://doi.org/10.2172/4419855.

[5] Dworkin, A. S.; Bredig, M. A. "The heat of fusion of the alkali metal halides." J. Phys. Chem. 1960, 64 (2), 269-272. https://doi.org/10.1021/j100831a023.

[6] Cantor, S.; Cooke, J. W.; Dworkin, A. S.; Robbins, G. D.; Thoma, R. E.; Watson, G. M. Physical properties of molten-salt reactor fuel, coolant, and flush salts. ORNL-TM-2316. Oak Ridge National Laboratory, 1968. https://doi.org/10.2172/4492893.

[7] Cohen, S. I.; Jones, T. N. A summary of density measurements on molten fluoride mixtures and a correlation for predicting densities of fluoride mixtures. ORNL-1702. Oak Ridge National Laboratory, 1954.

[8] Cohen, S. I.; Jones, T. N. Viscosity measurements on molten fluoride mixtures. ORNL-2278. Oak Ridge National Laboratory, 1957. https://doi.org/10.2172/4803933.

[9] Powers, W. D.; Cohen, S. I.; Greene, N. D. "Physical Properties of Molten Reactor Fuels and Coolants.” Nucl. Sci. Eng. 1963, 17 (2), 200-211. https://doi.org/10.13182/NSE63-5.

[10] Rose, M.; Wu, E.; Williamson, M. Thermophysical Property Measurements: Improved Density, Viscosity and Thermal Diffusivity Methods. ANL/CFCT-20/38164442. Argonne National Laboratory, 2020. https://doi.org/10.2172/1734862.

[11] Janz, G. J. "Thermodynamic and Transport Properties for Molten Salts: Correlation Equations for Critically Evaluated Density, Surface Tension, Electrical Conductance, and Viscosity Data." J. Phys. Chem. Ref. Data 1988, 17.

[12] Magnusson, J.; Memmott, M.; Munro, T. "Review of Thermophysical Property Methods Applied to Fueled and Un-Fueled Molten Salts." Ann. Nucl. Energy 2020, 146, 107608.

https://doi.org/10.1016/j.anucene.2020.107608.

[13] Williams, D. F. Assessment of Candidate Molten Salt Coolants for the Advanced High Temperature Reactor (AHTR). ORNL/TM-2006/12. Oak Ridge National Laboratory, 2006. https://doi.org/10.2172/885975.

[14] Williams, D. F. Assessment of Candidate Molten Salt Coolants for the NGNP/NHI Heat-Transfer Loop. ORNL/TM-2006/69. Oak Ridge National Laboratory, 2006. https://doi.org/10.2172/1360677.

[15] Jerden, J. Molten Salt Thermophysical Properties Database Development: 2019 Update. ANL/CFCT-19/6. Argonne National Laboratory, 2019. https://doi.org/10.2172/1559846. 
[16] Tasidou, K. A.; Chliatzou, C. D.; Assael, M. J.; Antoniadis, K. D.; Mylona, S. K.; Huber, M. L.; Wakeham, W. A. "Reference Correlations for the Viscosity of 13 Inorganic Molten Salts." $J$. Phys. Chem. Ref. Data 2019, 48 (1), 013101. https://doi.org/10.1063/1.5091511.

[17] Tasidou, K. A.; Magnusson, J.; Munro, T.; Assael, M. J. "Reference Correlations for the Viscosity of Molten LiF-NaF-KF, LiF-BeF 2 , and Li 2 CO 3 -Na 2 CO 3 -K 2 CO 3." J. Phys. Chem. Ref. Data 2019, 48 (4), 043102. https://doi.org/10.1063/1.5131349.

[18] Janz, G. J.; Dampier, F. W.; Lakshminarayanan, G. R.; Lorenz, P. K.; Tomkins, R. P. T. Molten Salts: Volume 1. Electrical Conductance, Density, and Viscosity Data (National Standard Reference Data Series-National Bureau of Standards 15; US Government Printing Office Washington, DC, 1968.

[19] Janz, G. J.; Gardner, G. L.; Krebs, U.; Tomkins, R. P. T. "Molten Salts: Volume 4, Part 1, Fluorides and Mixtures Electrical Conductance, Density, Viscosity, and Surface Tension Data." J. Phys. Chem. Ref. Data 1974, 3 (1), 1-115. https://doi.org/10.1063/1.3253134.

[20] Janz, G. J.; Tomkins, R. P. T.; Allen, C. B.; Downey, J. R.; Garner, G. L.; Krebs, U.; Singer, S. K. Molten Salts: "Volume 4, Part 2, Chlorides and Mixtures-Electrical Conductance, Density, Viscosity, and Surface Tension Data." J. Phys. Chem. Ref. Data 1975, 4 (4), 871-1178. https://doi.org/10.1063/1.555527.

[21] Janz, G. J.; Tomkins, R. P. T. Physical Properties Data Compilations Relevant to Energy Storage. IV. Molten Salts: Data on Additional Single and Multi-Component Salt Systems; National Standard Reference Data System, 1981.

[22] Janz, G. J.; Allen, C. B.; Bansal, N. P.; Murphy, R. M.; Tomkins, R. P. T. Physical Properties Data Compilations Relevant to Energy Storage. II. Molten Salts: Data on Single and MultiComponent Salt Systems; New York, 1979.

[23] Janz, G. J. Molten Salts Handbook; Elsevier: New York, 1967. https://doi.org/10.1016/B978-0123-95642-2.X5001-1.

[24] Serrano-López, R.; Fradera, J.; Cuesta-López, S. "Molten Salts Database for Energy Applications." Chem. Eng. Process. Process Intensif. 2013, 73, 87-102. https://doi.org/10.1016/j.cep.2013.07.008.

[25] McMurray, J. W.; Johnson, K.; Agca, C.; Betzler, B.; Kropaczek, D.; Besmann, T.; Andersson, D.; Ezell, N. D. Roadmap for Thermal Property Measurements of Molten Salt Reactor Systems. ORNL/SPR-2020/1865. Oak Ridge National Laboratory, 2021. https://doi.org/10.2172/1778081.

[26] Agca, C.; Johnson, K.; McMurray, J. W.; Yingling, J.; Besmann, T. FY21 Status Report on the Molten Salt Thermal Properties Database (MSTDB) Development. ORNL/SPR-2021/2102. Oak Ridge National Laboratory, 2021. https://doi.org/10.2172/1814280.

[27] Gheribi, A. E.; Torres, J. A.; Chartrand, P. "Recommended Values for the Thermal Conductivity of Molten Salts between the Melting and Boiling Points." Sol. Energy Mater. Sol. Cells 2014, 126, 11-25. https://doi.org/10.1016/j.solmat.2014.03.028.

[28] Nagasaka, Y.; Nakazawa, N.; Nagashima, A. "Experimental Determination of the Thermal Diffusivity of Molten Alkali Halides by the Forced Rayleigh Scattering Method. I. Molten LiCl, $\mathrm{NaCl}, \mathrm{KCl}, \mathrm{RbCl}$, and CsCl.” Int. J. Thermophys. 1992, 13 (4), 555-574. https://doi.org/10.1007/BF00501941.

[29] Ezell, N. D.; Gallagher, R.; Russell, N.; Martin, A.; Mcmurray, J.; McAlister, A. Thermophysical Property Measurements on Salt Mixture. ORNL/TM-2020/1633. Oak Ridge National Laboratory, 2020. https://doi.org/10.2172/1763479. 
[30] Agca, C.; McMurray, J. W. "Empirical Estimation of Densities in NaCl-KCl-UCl3 and NaClKCl-YCl3 Molten Salts Using Redlich-Kister Expansion.” Chem. Eng. Sci. 2021, 117086. https://doi.org/10.1016/j.ces.2021.117086.

[31] Redlich, O.; Kister, A. T. "Algebraic Representation of Thermodynamic Properties and the Classification of Solutions." Ind. Eng. Chem. 1948, 40 (2), 345-348.

[32] Arrhenius, S. Über Die Innere Reibung Verdünnter Wässeriger Lösungen. Zeitschrift für Phys. Chemie 1887, $1 U$ (1), 285-298. https://doi.org/10.1515/zpch-1887-0133.

[33] Dul'nev, G. N.; Zarichnyak, Y. P. “Thermal Conductivity of Liquid Mixtures.” J. Eng. Phys. 1966, 11 (6), 400-402. https://doi.org/10.1007/BF00829337.

[34] Kincaid, J. F.; Eyring, H. "Free Volumes and Free Angle Ratios of Molecules in Liquids." J. Chem. Phys. 1938, 6 (10), 620-629. https://doi.org/10.1063/1.1750134.

[35] Powell, R. E.; Roseveare, W. E.; Eyring, H. Diffusion, "Thermal Conductivity, and Viscous Flow of Liquids.” Ind. Eng. Chem. 1941, 33 (4), 430-435. https://doi.org/10.1021/ie50376a003.

[36] Mikami, M.; Odawara, O.; Kawamura, K. "Sound Velocity in Heat Transfer Salt (Mixture of Molten Sodium Nitrate-Potassium Nitrate-Sodium Nitrite, 7/44/49 Mol \%) Studied by an Ultrasonic Pulse-Echo Method.” J. Chem. Eng. Data 1981, 26 (4), 411-413.

https://doi.org/10.1021/je00026a019.

[37] McMurray, J. W.; Besmann, T. M.; Ard, J. C.; Utlak, S. A.; Lefebvre, J. P. Status of the Molten Salt Thermodynamic Database, MSTDB. ORNL/SPR-2019/1208. Oak Ridge National Laboratory, 2019.

[38] Muggianu, Y.-M.; Gambino, M.; Bros, J.-P. "Enthalpies de Formation Des Alliages Liquides Bismuth-Étain-Gallium à 723 k. Choix d'une Représentation Analytique Des Grandeurs d'excès Intégrales et Partielles de Mélange." J. Chim. Phys. 1975, 72, 83-88. https://doi.org/10.1051/jcp/1975720083.

[39] Hillert, M. "Empirical Methods of Predicting and Representing Thermodynamic Properties of Ternary Solution Phases." Calphad 1980, 4 (1), 1-12. https://doi.org/10.1016/03645916(80)90016-4. 


\section{APPENDIX A. MOLTEN SALT THERMOPHYISCAL DATA}



APPENDIX A. MOLTEN SALT THERMOPHYISCAL DATA

\begin{tabular}{|c|c|c|c|c|c|c|c|c|c|c|c|c|c|c|c|c|c|c|c|c|c|c|c|c|c|c|c|c|c|c|c|c|}
\hline \multirow{2}{*}{\multicolumn{2}{|c|}{ 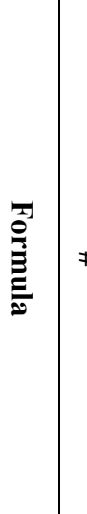 }} & \multirow[t]{2}{*}{ 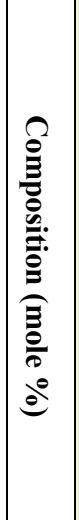 } & \multirow[t]{2}{*}{ 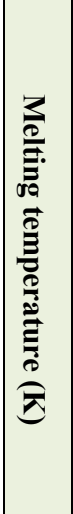 } & \multirow[t]{2}{*}{ 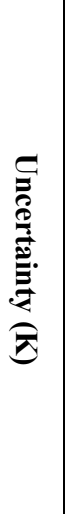 } & \multirow[t]{2}{*}{ 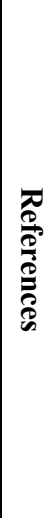 } & \multirow[t]{2}{*}{ 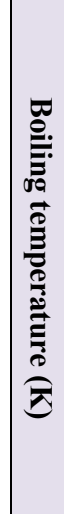 } & \multirow[t]{2}{*}{ 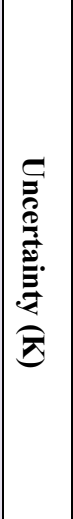 } & \multirow[t]{2}{*}{ 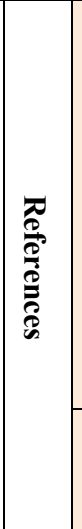 } & \multicolumn{2}{|c|}{ 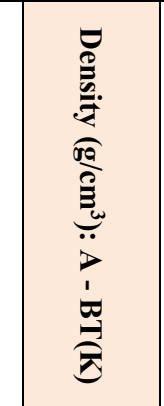 } & \multirow{2}{*}{ 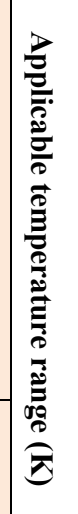 } & \multirow[t]{2}{*}{ 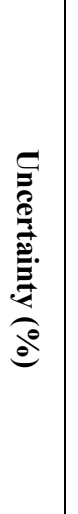 } & \multirow[t]{2}{*}{ 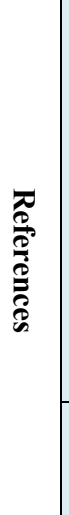 } & \multicolumn{2}{|c|}{ 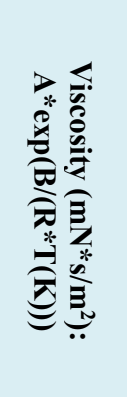 } & \multicolumn{3}{|c|}{ 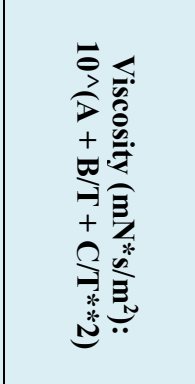 } & \multirow{2}{*}{ 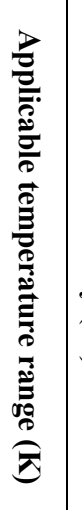 } & \multirow[t]{2}{*}{ 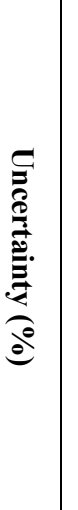 } & \multirow[t]{2}{*}{ 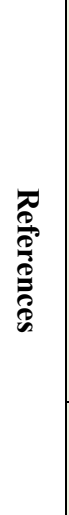 } & \multicolumn{2}{|c|}{ 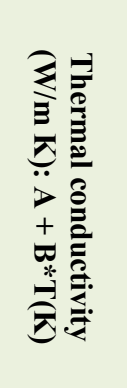 } & \multirow[t]{2}{*}{ 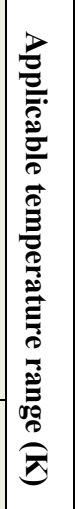 } & \multirow[t]{2}{*}{ 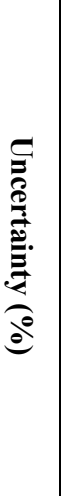 } & \multicolumn{5}{|c|}{ 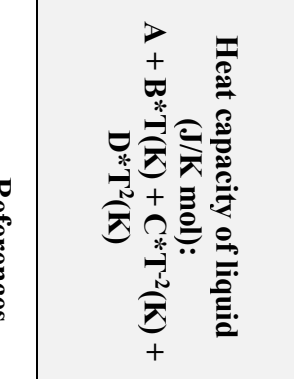 } & \multirow[t]{2}{*}{ 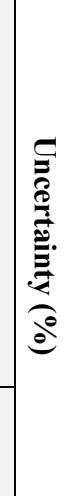 } & \multirow[t]{2}{*}{ 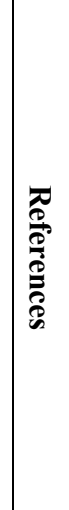 } \\
\hline & & & & & & & & & $>$ & $\varpi$ & & & & $>$ & $\nabla$ & $>$ & $\varpi$ & 0 & & & & $>$ & $\varpi$ & & & & $>$ & $\sigma$ & $\Omega$ & $\theta$ & & \\
\hline${ }_{\mathrm{r}}$ & - & 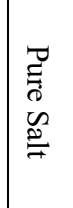 & 吾 & 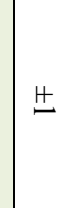 & 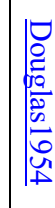 & . & | & 曐 & 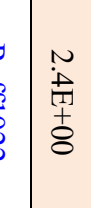 & 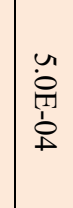 & 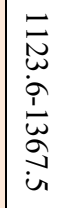 & $\Delta$ & 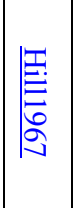 & $\begin{array}{l}\dot{\vec{T}} \\
\dot{\omega}\end{array}$ & $\mid \begin{array}{l}n \\
y \\
\text { y } \\
+ \\
+\end{array}$ & 1 & | & 1 & 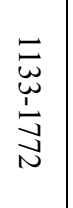 & $\Delta$ & 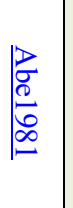 & 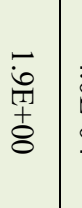 & 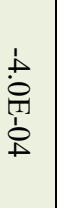 & $\begin{array}{l}\overrightarrow{\vec{D}} \\
0 \\
\dot{1} \\
\overline{0} \\
\dot{8}\end{array}$ & $\tilde{\sigma}$ & 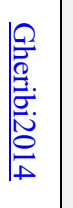 & $\begin{array}{l}\text { ì } \\
\text { 龺 }\end{array}$ & | & | & 1 & $\vec{\omega}$ & 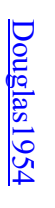 \\
\hline 㤣 & N & 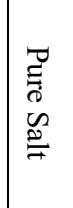 & $\begin{array}{l}\vec{\Omega} \\
\infty \\
0 \\
0\end{array}$ & 草 & 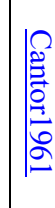 & $\mid \begin{array}{l}\overrightarrow{0} \\
0 \\
0 \\
0\end{array}$ & | & 曐 & 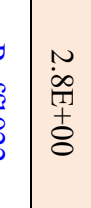 & $\begin{array}{l}\dot{f} \\
\dot{f} \\
\dot{b} \\
\dot{f}\end{array}$ & 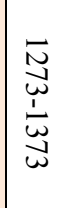 & i & 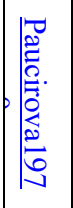 & $\begin{array}{l}\vec{i} \\
\text { Ty } \\
\text { वे }\end{array}$ & 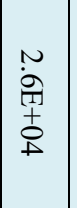 & 1 & 1 & 1 & $\begin{array}{l}\overline{\vec{N}} \\
\vec{U} \\
\underline{\omega} \\
\vec{\omega} \\
\end{array}$ & - & 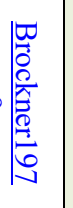 & 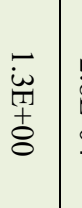 & $\begin{array}{l}\dot{n} \\
\infty \\
0 \\
1 \\
1 \\
\vdots\end{array}$ & 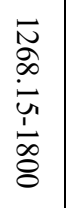 & $\tilde{\sigma}$ & 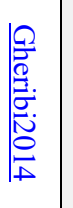 & 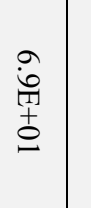 & | & | & | & $\because$ & 递 \\
\hline 盯 & $\omega$ & 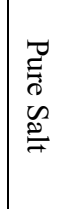 & $\stackrel{\vec{i}}{\vec{\omega}}^{\vec{n}}$ & 莒 & 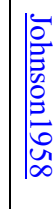 & : & & 萑 & $\begin{array}{l}n \\
\tilde{a} \\
\dot{T} \\
8 \\
\delta\end{array}$ & $\begin{array}{l}\dot{a} \\
\dot{y} \\
\dot{p} \\
\dot{p}\end{array}$ & 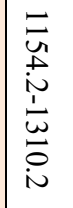 & $\Delta$ & 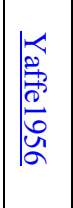 & $\begin{array}{l}\overrightarrow{\dot{\bar{T}}} \\
\dot{\omega}\end{array}$ & $\left|\begin{array}{l}N \\
f \\
+1 \\
+ \\
+\end{array}\right|$ & 1 & | & | & 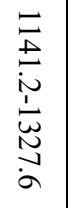 & i & 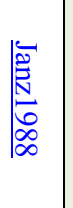 & 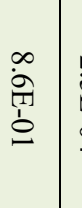 & 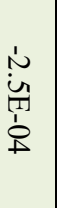 & 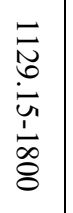 & $\tilde{o}$ & 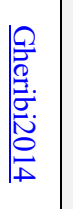 & $\begin{array}{l}\overrightarrow{\vec{T}} \\
\text { 莫 }\end{array}$ & | & | & 1 & $\vec{\infty}$ & 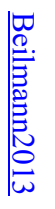 \\
\hline 桑 & + & 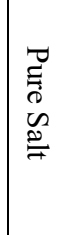 & 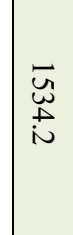 & 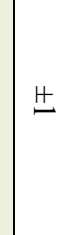 & 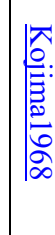 & $\mid \begin{array}{l}\tilde{N} \\
\tilde{N} \\
\end{array}$ & & 疍 & 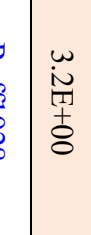 & 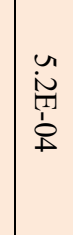 & 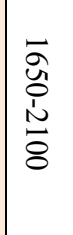 & $\ddot{9}$ & 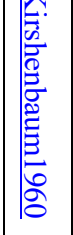 & $\begin{array}{l}\overrightarrow{0} \\
\text { T⿱10} \\
\dot{0}\end{array}$ & 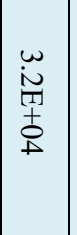 & 1 & | & | & 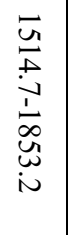 & $\omega$ & 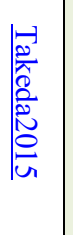 & $\begin{array}{l}\text { 茪 } \\
\dot{\leftrightarrow}\end{array}$ & $\begin{array}{l}+1 \\
\dot{1} \\
\dot{+}\end{array}$ & 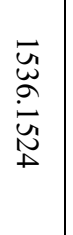 & $\tilde{\sigma}$ & 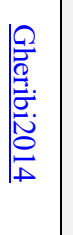 & 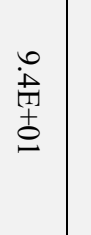 & | & | & | & i & 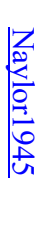 \\
\hline
\end{tabular}




\begin{tabular}{|c|c|c|c|c|c|c|c|c|c|c|c|c|c|c|c|c|c|c|c|c|c|c|c|c|c|c|c|c|c|c|c|}
\hline 慰 & ur & 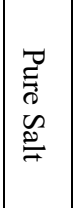 & 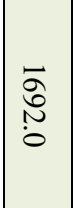 & $\stackrel{\Perp}{*}$ & 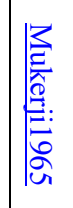 & \begin{tabular}{|l}
$\mathbf{N}$ \\
$\mathbb{N}$ \\
+
\end{tabular} & $\mid$ & 萑 & 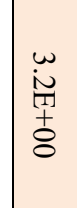 & 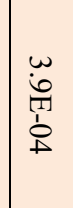 & 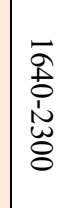 & $\stackrel{\circ}{\dot{9}}$ & 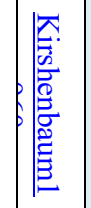 & $\begin{array}{l}\overrightarrow{\dot{\hat{\varphi}}} \\
\dot{\omega} \\
\dot{\omega}\end{array}$ & 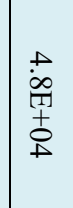 & 1 & 1 & 1 & 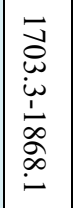 & $\omega$ & 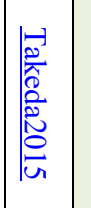 & 离 & $\begin{array}{l}1 \\
\dot{\hat{T}} \\
\dot{1} \\
\dot{u}\end{array}$ & 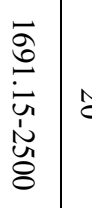 & $\approx \mid$ & 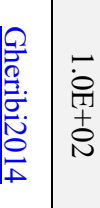 & 1 & 1 & 1 & $\overline{\mathrm{N}}$ & 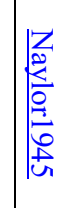 \\
\hline 等 & $a$ & 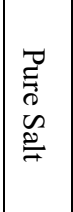 & $\begin{array}{l}\overrightarrow{\vec{T}} \\
\dot{A} \\
0\end{array}$ & 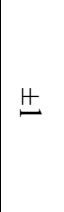 & 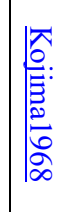 & 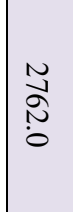 & 1 & 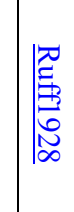 & 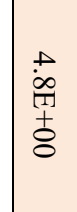 & \begin{tabular}{|l}
$\vec{y}$ \\
$\dot{y}$ \\
$\dot{1}$ \\
$\dot{y}$
\end{tabular} & 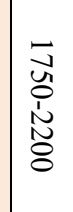 & $\dot{\dot{9}}$ & 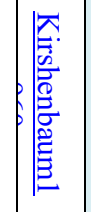 & 帘 & 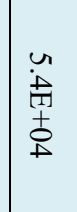 & 1 & 1 & 1 & $\mid$\begin{tabular}{l|}
$\vec{a}$ \\
+ \\
+ \\
+ \\
$+\infty$ \\
$\infty$ \\
$+\infty$ \\
$\dot{0}$
\end{tabular} & $\omega$ & 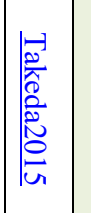 & $\mid \begin{array}{c}\tilde{u} \\
\tilde{T} \\
\dot{1} \\
\underline{v}\end{array}$ & 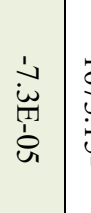 & $\begin{array}{l}\overrightarrow{\widehat{ज}} \\
\overrightarrow{\bar{c}}\end{array}$ & $\approx$ & 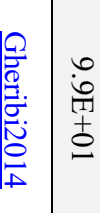 & | & 1 & 1 & $\mid 1$ & 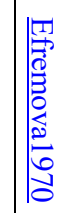 \\
\hline 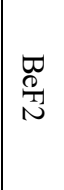 & $\checkmark$ & 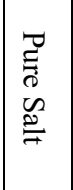 & $\frac{\infty}{\stackrel{N}{N}}$ & $\stackrel{\Perp}{ }$ & 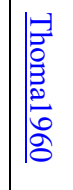 & 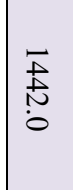 & 1 & 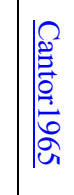 & 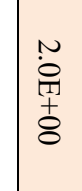 & \begin{tabular}{|l}
$\overline{\dot{y}}$ \\
$\dot{\bar{\varphi}}$ \\
$\dot{u}$
\end{tabular} & 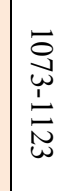 & in & 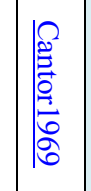 & 1 & 1 & 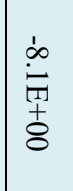 & 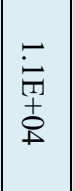 & 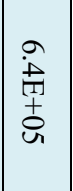 & 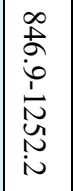 & $\omega$ & 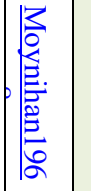 & \begin{tabular}{|l|}
$\infty$ \\
$\dot{0}$ \\
$\dot{1}$ \\
0 \\
0
\end{tabular} & 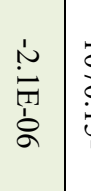 & 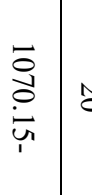 & $\tilde{\sigma}$ & 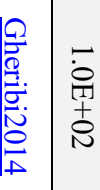 & $\begin{array}{l}\frac{1}{\dot{\omega}} \\
\frac{\hat{T}}{1} \\
\dot{\omega}\end{array}$ & 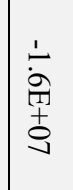 & $\begin{array}{l}\omega \\
\dot{0} \\
\dot{1} \\
\dot{0}\end{array}$ & 1 & 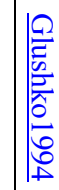 \\
\hline 壳 & $\infty$ & 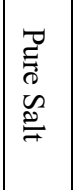 & $\begin{array}{l}\overrightarrow{\vec{J}} \\
\infty \\
i \\
i\end{array}$ & 芯 & 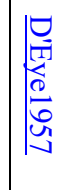 & $\begin{array}{l}\tilde{u} \\
\breve{d} \\
\dot{0}\end{array}$ & $\mid$ & 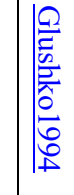 & 1 & 1 & 1 & | & 1 & 1 & 1 & 1 & 1 & $\mid$ & 1 & $\mid$ & 1 & 1 & & | & | & 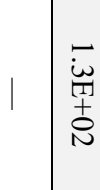 & 1 & 1 & 1 & un & 旡 \\
\hline 蛋 & 6 & 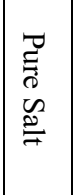 & \begin{tabular}{|l|}
$\overline{\breve{\omega}}$ \\
$\dot{0}$ \\
0
\end{tabular} & 先 & 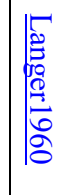 & $\mid \begin{array}{c}\vec{N} \\
0 \\
0\end{array}$ & 1 & 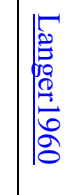 & 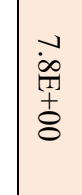 & $\begin{array}{l}0 \\
\dot{0} \\
\dot{10} \\
\dot{1} \\
\dot{+}\end{array}$ & 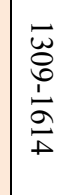 & $\Delta$ & 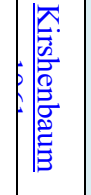 & 1 & 1 & \begin{tabular}{|l}
$\overrightarrow{\bar{m}}$ \\
草
\end{tabular} & 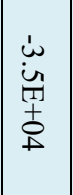 & 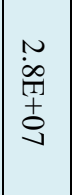 & \begin{tabular}{|l|}
$\overrightarrow{\breve{w}}$ \\
$\infty$ \\
$\stackrel{\infty}{\sigma}$ \\
$\stackrel{\infty}{\infty}$
\end{tabular} & o & 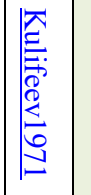 & 1 & & I & I & | $\mid$\begin{tabular}{l|l} 
\\
|
\end{tabular} & 1 & 1 & 1 & & 离 \\
\hline 悹 & $\bar{o}$ & 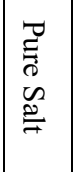 & $\mid$\begin{tabular}{l}
$\vec{u}$ \\
\hdashline \\
0
\end{tabular} & 芯 & 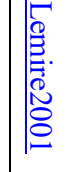 & $\begin{array}{l}\tilde{\breve{y}} \\
\stackrel{0}{0}\end{array}$ & 1 & $\mid$ & 1 & 1 & 1 & | & 1 & 1 & 1 & 1 & 1 & 11 & $\mid$ & 1 & $\mid$ & 1 & & I & | & 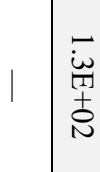 & 1 & 1 & 1 & $\vec{u}$ & : \\
\hline 芯 & $=$ & 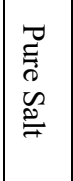 & $\mid \begin{array}{l}\overrightarrow{0} \\
0 \\
0 \\
0\end{array}$ & 笖 & 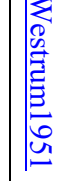 & 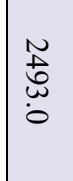 & . & | & 1 & 1 & 1 & | & 1 & 1 & 1 & 1 & 1 & 1 & $\mid$ & 1 & $\mid$ & 1 & & | & | & 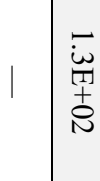 & 1 & 1 & 1 & $u_{r}$ & $\vec{k}$ \\
\hline
\end{tabular}




\begin{tabular}{|c|c|c|c|c|c|c|c|c|c|c|c|c|c|c|c|c|c|c|c|c|c|c|c|c|c|c|c|c|c|c|c|}
\hline 产 & $\bar{N}$ & 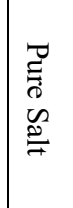 & $\begin{array}{l}\bar{\sigma} \\
\text { ôे }\end{array}$ & 芯 & 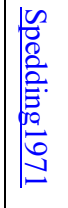 & $\begin{array}{l}\tilde{N} \\
\text { oे } \\
0 \\
0\end{array}$ & 1 & $\mid$ & $\begin{array}{l}\text { w } \\
0 \\
0 \\
0 \\
8\end{array}$ & $\begin{array}{l}a \\
0 \\
0 \\
\dot{1} \\
\dot{p}\end{array}$ & 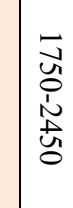 & $\stackrel{0}{\dot{v}}$ & 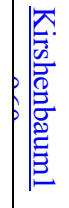 & । & 1 & 1 & 1 & | & $\mid$ & $\mid$ & $\mid$ & I & & 1 11 & 101 & 离 & 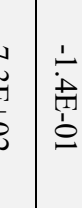 & {$\left[\begin{array}{l}t \\
w \\
w \\
w \\
\infty \\
\infty\end{array}\right.$} & $\mid$ & $\omega$ & 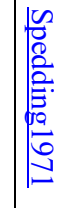 \\
\hline 总 & $\bar{\omega}$ & 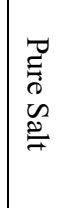 & $\begin{array}{l}\overline{\mathrm{d}} \\
\text { Oे }\end{array}$ & 出 & 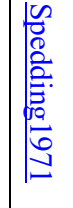 & 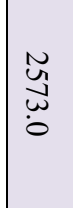 & 1 & 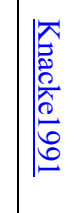 & 1 & | & $\mid$ & $\mid$ & 1 & 1 & 1 & 1 & 1 & | & 1 & 1 & $\mid$ & | & & \begin{tabular}{l|l}
1 & 1
\end{tabular} & 1 11 & 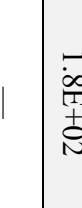 & 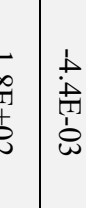 & 离 & | 1 & $\omega$ & 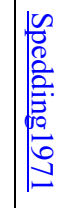 \\
\hline 鳃 & $F$ & 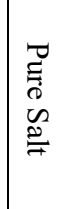 & 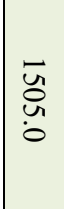 & H & 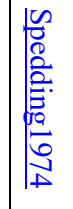 & $\begin{array}{l}\tilde{\breve{u}} \\
\stackrel{d}{0}\end{array}$ & 1 & 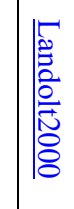 & 1 & | & | & $\mid$ & 1 & 1 & 1 & 1 & 1 & $\mid$ & 1 & 1 & $\mid$ & I & & | & | & 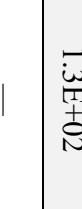 & 葡 & | & | 1 & $\omega$ & 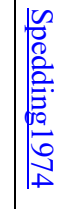 \\
\hline$\stackrel{5}{\Xi}$ & $\vec{u}$ & 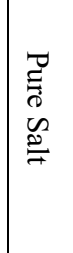 & 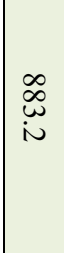 & 先 & 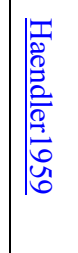 & $\begin{array}{l}\overline{\bar{u}} \\
0 \\
0\end{array}$ & 1 & 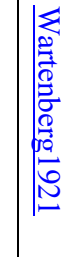 & 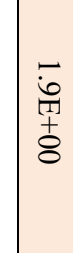 & 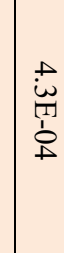 & 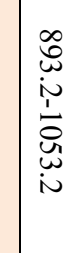 & $\Delta$ & 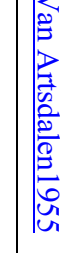 & $\begin{array}{l}\vec{w} \\
\vec{w} \\
\dot{w} \\
\dot{w}\end{array}$ & 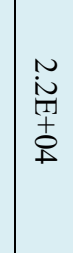 & 1 & 1 & 1 & 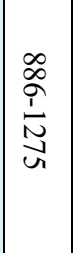 & N & 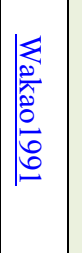 & $\begin{array}{l}\infty \\
\infty \\
0 \\
0 \\
\stackrel{1}{6}\end{array}$ & 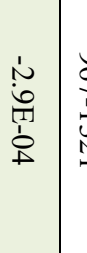 & 荢 & $\mathbb{0}$ & 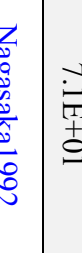 & 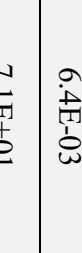 & | & 1 & in & 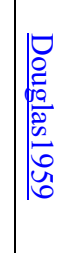 \\
\hline Zू̆ & $\bar{a}$ & 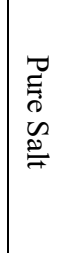 & $\mid \begin{array}{c}\overline{0} \\
\dot{\omega} \\
\infty\end{array}$ & $\stackrel{\Perp}{ \pm}$ & 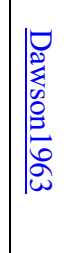 & $\begin{array}{l}\overrightarrow{\vec{u}} \\
0 \\
0\end{array}$ & 1 & 苗 & 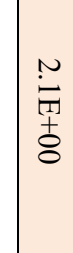 & 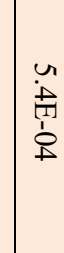 & 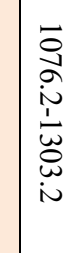 & $\Delta$ & 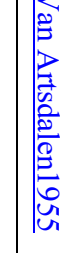 & $\begin{array}{l}\infty \\
0 \\
0 \\
\text { ஸे } \\
\text { in }\end{array}$ & 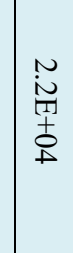 & 1 & 1 & | & 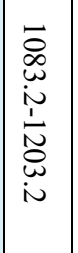 & $\Delta$ & 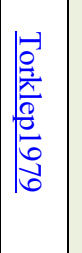 & $\begin{array}{l}\text { 苛 } \\
\stackrel{0}{\circ}\end{array}$ & 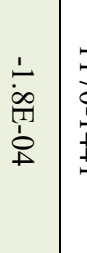 & 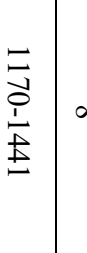 & $\infty$ & 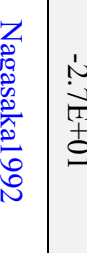 & 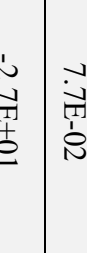 & 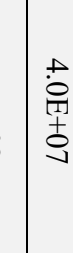 & $\begin{array}{l}\frac{1}{\omega} \\
0 \\
\dot{0} \\
\dot{\omega}\end{array}$ & $\ddot{\omega}$ & 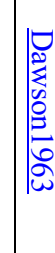 \\
\hline 至 & $\vec{v}$ & $\begin{array}{l}\vec{J} \\
\underline{E} \\
w \\
\tilde{E}\end{array}$ & 总 & 嚄 & 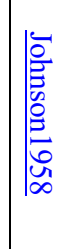 & $\begin{array}{l}\bar{\partial} \\
\infty \\
0 \\
0\end{array}$ & $\mid$ & 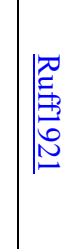 & $\begin{array}{l}\mathrm{N} \\
\vec{T} \\
\mathbf{8}\end{array}$ & $\begin{array}{l}0 \\
0 \\
0 \\
\vdots \\
\vdots \\
\vdots\end{array}$ & 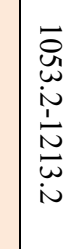 & $\Delta$ & 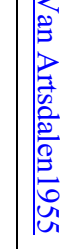 & $\begin{array}{l}\overrightarrow{\dot{T}} \\
\text { 崇 }\end{array}$ & 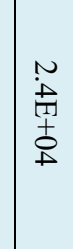 & 1 & 1 & 1 & 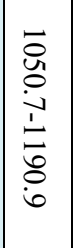 & $\Delta$ & 震: & $\begin{array}{l}\dddot{u} \\
\ddot{T} \\
\stackrel{1}{o}\end{array}$ & 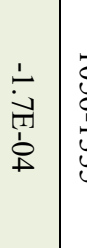 & 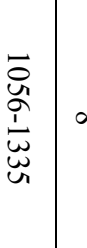 & $\infty$ & 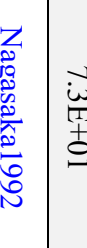 & | & I & 1 & N & 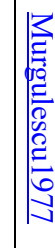 \\
\hline
\end{tabular}




\begin{tabular}{|c|c|c|c|c|c|c|c|c|c|c|c|c|c|c|c|c|c|c|c|c|c|c|c|c|c|c|c|c|c|c|}
\hline $\begin{array}{l}\frac{3}{80} \\
\frac{a}{n} \\
\frac{0}{n}\end{array}$ & $\vec{\infty}$ & 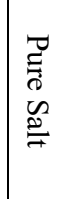 & 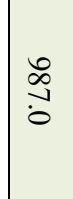 & \pm & 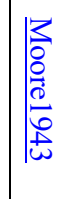 & $\begin{array}{l}\bar{a} \\
\text { d़े. } \\
0\end{array}$ & 莕 & 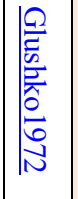 & \begin{tabular}{|l|} 
\\
$\dot{0}$ \\
章 \\
8
\end{tabular} & $\begin{array}{l}\dot{w} \\
\dot{\omega} \\
\dot{\omega} \\
\dot{\phi}\end{array}$ & 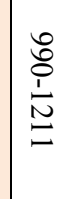 & - & 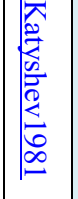 & 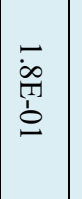 & 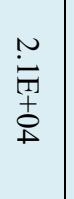 & | & | & 1 & - & 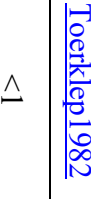 & 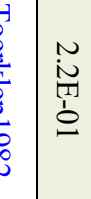 & $\mid \begin{array}{l}\dot{1} \\
\tilde{O} \\
\hat{T} \\
\dot{o} \\
u\end{array}$ & 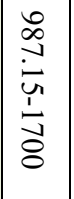 & No & 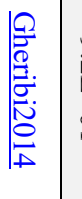 & 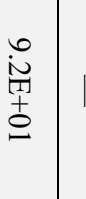 & | & | & & $\Delta$ \\
\hline 苂 & 6 & 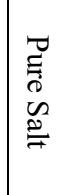 & 宓 & 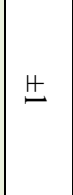 & $\mid \begin{array}{l}\mid z \\
0 \\
0 \\
0 \\
0 \\
0 \\
0 \\
0\end{array}$ & \begin{tabular}{|l}
$\mathbb{N}$ \\
U. \\
0
\end{tabular} & $\underset{\sim}{\stackrel{H}{*}}$ & 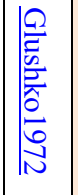 & 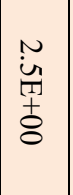 & $\begin{array}{l}\text { 蒂 } \\
\dot{1} \\
\dot{t}\end{array}$ & 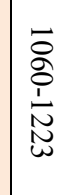 & $\Delta$ & 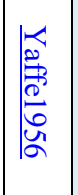 & 1 & 1 & $\begin{array}{l}\dot{u} \\
\dot{y} \\
\dot{1} \\
\dot{\theta}\end{array}$ & 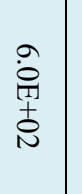 & 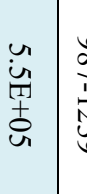 & 总 & 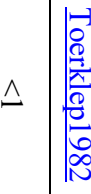 & 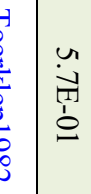 & 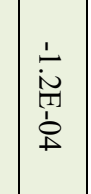 & 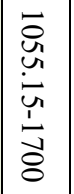 & $\tilde{\sigma}$ & 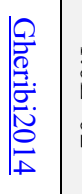 & 窝 & | & | & | & $\Delta$ \\
\hline$\frac{n}{2}$ & ro & 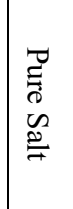 & 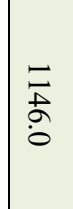 & 总 & 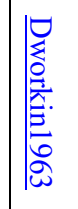 & \begin{tabular}{|l}
$\tilde{W}$ \\
$\tilde{O}$ \\
0
\end{tabular} & 苔 & 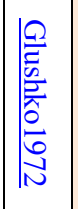 & 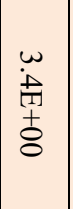 & 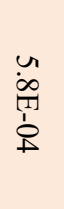 & 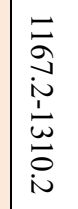 & $\Delta$ & 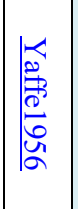 & 1 & 1 & 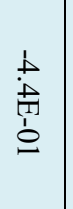 & $\begin{array}{l}\text { 龺 } \\
\text { 索 }\end{array}$ & 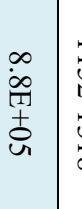 & 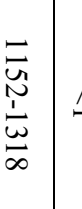 & 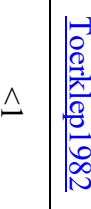 & 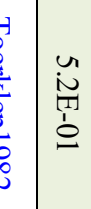 & 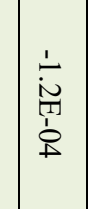 & 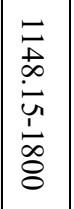 & $\tilde{\sigma}$ & 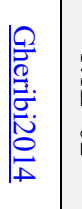 & 离 & | & | & | & \\
\hline $\begin{array}{l}\text { 品 } \\
\stackrel{2}{\bar{\nu}}\end{array}$ & $\simeq$ & 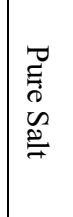 & $\begin{array}{l}\stackrel{2}{\infty} \\
\infty \\
\infty\end{array}$ & 点 & 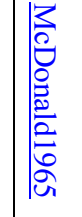 & वे & 1 & 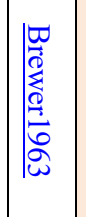 & \begin{tabular}{|l|} 
\\
$w$ \\
$w$ \\
$w$ \\
\\
8 \\
\end{tabular} & $\begin{array}{l}\overrightarrow{\bar{T}} \\
\dot{\omega} \\
\dot{\omega}\end{array}$ & 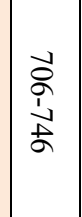 & $N$ & 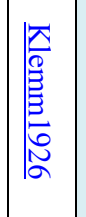 & 1 & I & 1 & 1 & | & | & $1 \mid 1$ & $\mid$ & & 1 & 1 & | & 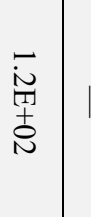 & | & | & | & $\Delta$ \\
\hline 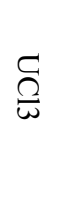 & N & 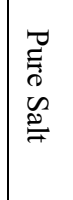 & $\vec{\Xi}$ & \pm & 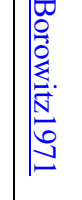 & 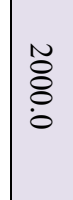 & 1 & 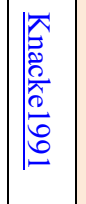 & 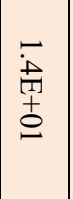 & $\begin{array}{l}\vec{b} \\
\text { 崩 } \\
\dot{\omega}\end{array}$ & 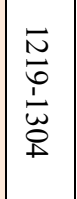 & $\overrightarrow{i_{n}}$ & 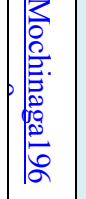 & 1 & 1 & 1 & I & I & 1 & | & $\mid$ & & 1 & I & I & 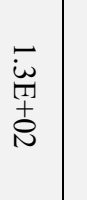 & | & | & | & | \\
\hline$\frac{z}{z}$ & N & 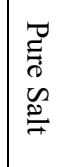 & في & $\underset{0}{\Delta}$ & 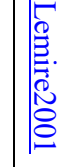 & $\begin{array}{l}\infty \\
\stackrel{\infty}{0} \\
\stackrel{0}{0}\end{array}$ & 1 & 宸 & 1 & 1 & 1 & 1 & 1 & 1 & 1 & 1 & I & I & 1 & | & | & & 1 & 1 & | & 謍 & | & | & | & $=$ \\
\hline 芯 & $\stackrel{N}{\perp}$ & 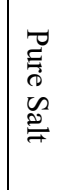 & 蓭 & 惫 & 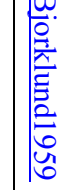 & 芯 & 1 & 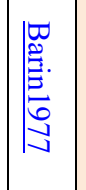 & 1 & 1 & 1 & 1 & 1 & 1 & 1 & 1 & I & 1 & 1 & | & 1 & & 1 & 1 & | & 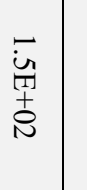 & | & | & | & 6 \\
\hline
\end{tabular}




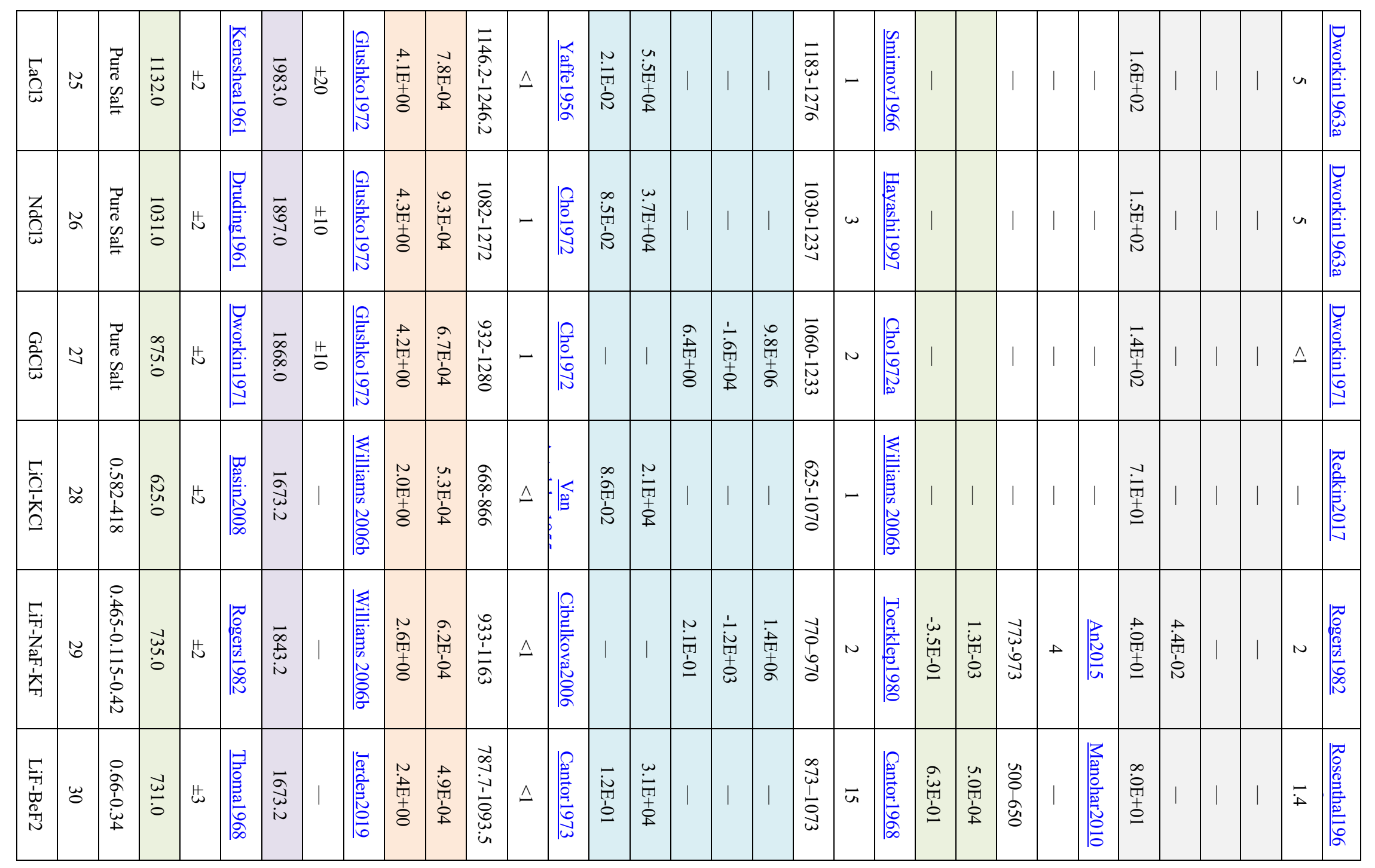




\begin{tabular}{|c|c|c|c|c|c|c|c|c|c|c|c|c|c|c|c|c|c|c|c|c|c|c|c|c|c|c|c|c|c|c|c|}
\hline 3 & $\stackrel{\omega}{-}$ & $\begin{array}{l}\dot{P} \\
\dot{\hat{\phi}} \\
\dot{\phi} \\
\dot{i}\end{array}$ & 家 & 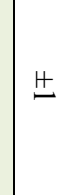 & 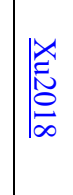 & $\frac{v}{\mathrm{~V}}$ & $\mid$ & 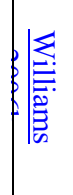 & 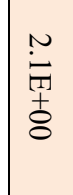 & $\begin{array}{l}u \\
\ddot{u} \\
\grave{1} \\
\dot{b}\end{array}$ & 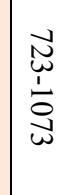 & $\overline{\mathrm{i}}$ & 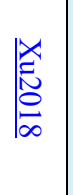 & I & 1 & 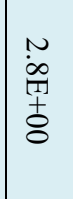 & 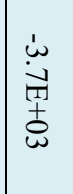 & \begin{tabular}{|l}
$\overrightarrow{\bar{y}}$ \\
索 \\
a
\end{tabular} & 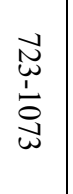 & $\checkmark$ & 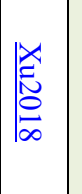 & 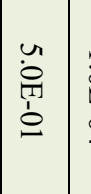 & 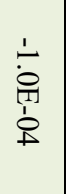 & 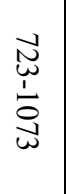 & $\checkmark$ & 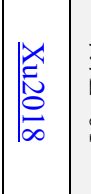 & $\begin{array}{l}\vec{y} \\
\text { 辛 } \\
0\end{array}$ & 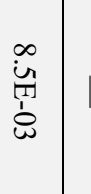 & \begin{tabular}{l|l}
1 & 1
\end{tabular} & | $\mid u$ & 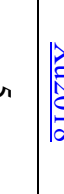 \\
\hline 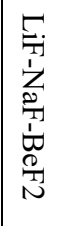 & $\underset{N}{\sim}$ & 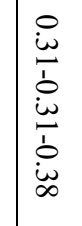 & $\mid \begin{array}{c}\text { మ } \\
\infty \\
0\end{array}$ & | & 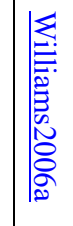 & 1 & 1 & | & 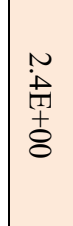 & $\begin{array}{l}+ \\
0 \\
010 \\
1 \\
\vdots\end{array}$ & 1 & 1 & 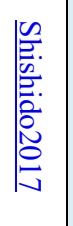 & 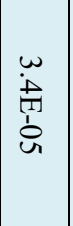 & 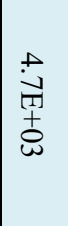 & 1 & 1 & 1 & | & & 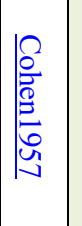 & \begin{tabular}{|l}
$\mid \overrightarrow{0}$ \\
苞
\end{tabular} & & 1 & 1 & 1 & $\begin{array}{l}\text { 卤 } \\
\dot{1} \\
\dot{c}\end{array}$ & I & | & 1 11 & | \\
\hline 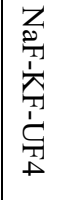 & $\stackrel{\omega}{\omega}$ & 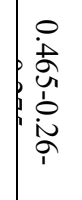 & 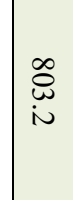 & | & 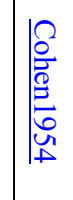 & 1 & 1 & | & 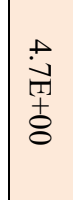 & 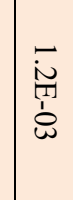 & 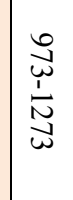 & $\omega$ & 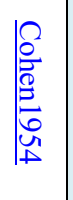 & 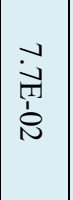 & 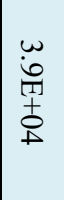 & 1 & 1 & 1 & 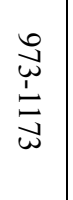 & $\overline{0}$ & 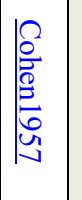 & 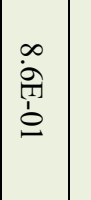 & I & I & $\tilde{\sim}$ & $\mid \begin{array}{l}0 \\
0 \\
0 \\
0 \\
0 \\
0 \\
0 \\
0 \\
0\end{array}$ & $\begin{array}{l}\text { 离 } \\
\text { 㕝 }\end{array}$ & | & \begin{tabular}{l|l}
1 & 1
\end{tabular} & $1 \mid \sigma$ & 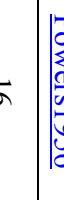 \\
\hline 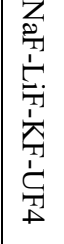 & $\underset{\leftarrow}{\Psi}$ & 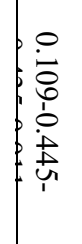 & 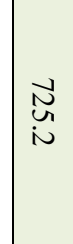 & | & 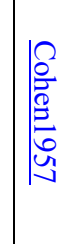 & 1 & 1 & | & 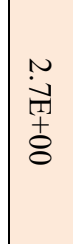 & $\begin{array}{l}0 \\
\dot{0} \\
\dot{1} \\
\dot{1} \\
\dot{f}\end{array}$ & 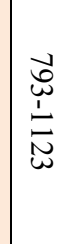 & $\omega$ & 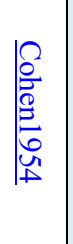 & 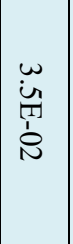 & 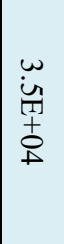 & 1 & 1 & 1 & $\begin{array}{l}\vec{u} \\
\vec{u} \\
\stackrel{0}{0} \\
\vec{\omega}\end{array}$ & o & 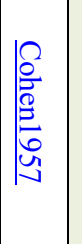 & \begin{tabular}{|l}
+ \\
$\dot{0}$ \\
T⿱宀 \\
$\dot{1}$ \\
$\dot{8}$
\end{tabular} & 1 & | & $\tilde{M}$ & 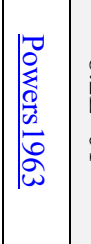 & 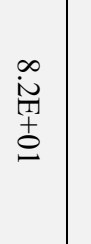 & I & | & $1 \mid \sigma$ & 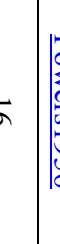 \\
\hline 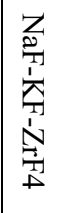 & $u_{u}$ & 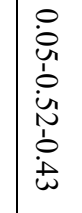 & 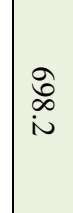 & | & 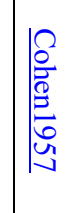 & 1 & $\mid$ & 1 & 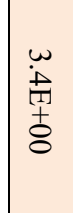 & 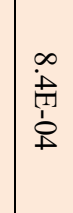 & | & 1 & 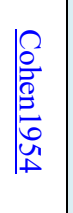 & $\begin{array}{l}\overrightarrow{\dot{\omega}} \\
\dot{\vec{\omega}} \\
\dot{b}\end{array}$ & 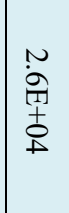 & 1 & 1 & 1 & $\mid \begin{array}{l}\vec{y} \\
\vdots \\
\vdots \\
\bar{\omega} \\
\omega\end{array}$ & $\overrightarrow{0}$ & 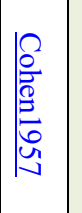 & 1 & I & | & | & 1 & 1 & I & | & 1 11 & 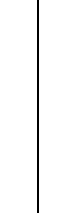 \\
\hline 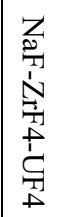 & w & 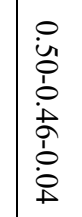 & 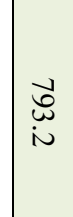 & | & 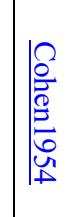 & 1 & $\mid$ & 1 & $\begin{array}{l}0 \\
\text { i } \\
\text { T1 } \\
\text { 8 }\end{array}$ & 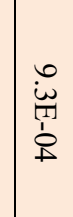 & | & 1 & 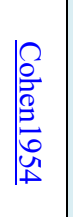 & 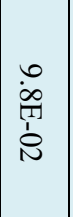 & 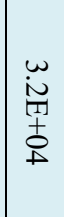 & 1 & 1 & 1 & 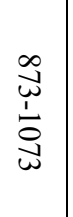 & $a$ & 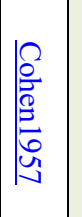 & 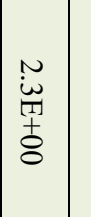 & I & I & 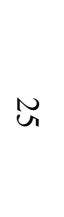 & $\mid$\begin{tabular}{|l}
0 \\
0 \\
0 \\
0 \\
0 \\
0 \\
0 \\
0 \\
0 \\
0
\end{tabular} & 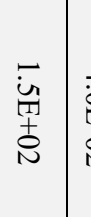 & $\begin{array}{l}\text { 南 } \\
\text { 商 }\end{array}$ & | & 10 & 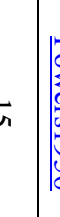 \\
\hline
\end{tabular}




\begin{tabular}{|c|c|c|c|c|c|c|c|c|c|c|c|c|c|c|c|c|c|c|c|c|c|c|c|c|c|c|c|c|c|c|c|}
\hline 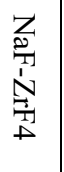 & $\stackrel{\sim}{\sim}$ & $\begin{array}{l}0 \\
\dot{y} \\
i \\
\dot{i} \\
\dot{y} \\
0\end{array}$ & $\stackrel{\vec{w}}{\stackrel{\infty}{\sim}}$ & | & 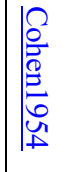 & 1 & | & | & 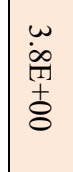 & \begin{tabular}{|l}
$\dot{0}$ \\
$\dot{w}$ \\
$\dot{\omega}$ \\
$\dot{1}$ \\
$\dot{+}$
\end{tabular} & 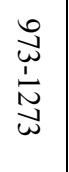 & $\omega$ & 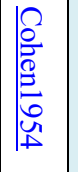 & 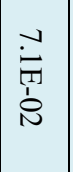 & $\begin{array}{l}w \\
\dot{u} \\
\text { 㕝 } \\
\end{array}$ & | & I & 1 & 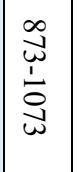 & 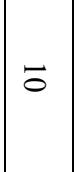 & 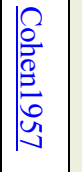 & | & 1 & $1 \mid 1$ & | & 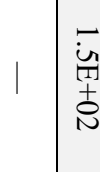 & 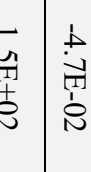 & | & 1 & $\vec{n}$ & $\begin{array}{l}0 \\
0 \\
0 \\
0 \\
0 \\
0 \\
0 \\
0 \\
0\end{array}$ \\
\hline 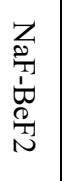 & $\underset{\infty}{\infty}$ & $\begin{array}{l}0 \\
\dot{i} \\
\hat{1} \\
\dot{t} \\
\dot{\omega}\end{array} \mid$ & $\underset{\text { 岕 }}{\stackrel{Q}{u}}$ & I & 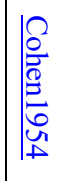 & $\underset{\substack{\vec{\sim} \\
i}}{\overrightarrow{\vec{v}}}$ & I & 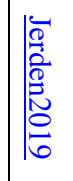 & 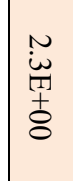 & 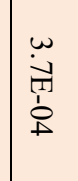 & 1 & I & 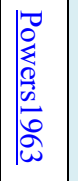 & 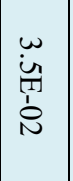 & 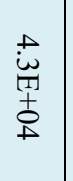 & | & I & 1 & 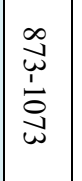 & $a$ & 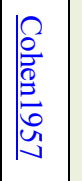 & 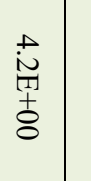 & | & 100 & ü & 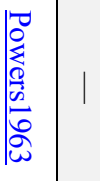 & \begin{tabular}{l|l}
1 & 1
\end{tabular} & | & 1 & 1 & | \\
\hline 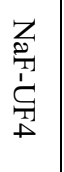 & $\omega$ & $\begin{array}{l}0 \\
\dot{a} \\
\hat{j} \\
\dot{\omega} \\
\dot{\omega} \\
\omega\end{array}$ & 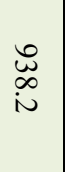 & 1 & 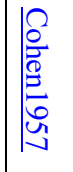 & 1 & | & | & $\begin{array}{l}\text { u } \\
\text { ur } \\
\text { 辛 }\end{array}$ & \begin{tabular}{|l}
$\overline{\dot{\omega}}$ \\
$\dot{\omega}$ \\
$\dot{\omega}$
\end{tabular} & 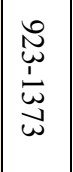 & $\omega$ & 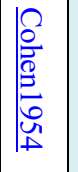 & 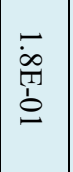 & 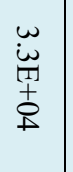 & | & | & 1 & 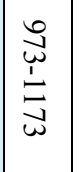 & $\overline{0}$ & 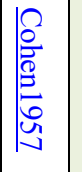 & I & 1 & $1 \mid 1$ & | & | & \begin{tabular}{l|l}
1 & 1
\end{tabular} & | & 1 & 1 & | \\
\hline 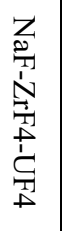 & 8 & 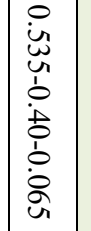 & $\varliminf_{i}^{\infty}$ & 1 & $\mid \begin{array}{l}0 \\
\frac{0}{0} \\
0 \\
0 \\
0 \\
0 \\
y \\
y\end{array}$ & 1 & | & | & 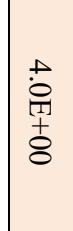 & \begin{tabular}{|l|}
$\overrightarrow{\dot{\bar{t}}}$ \\
$\dot{\omega}$ \\
$\dot{\omega}$
\end{tabular} & 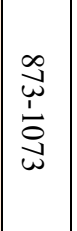 & $\omega$ & 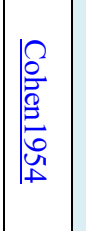 & $\begin{array}{l}\overrightarrow{\dot{b}} \\
\stackrel{1}{1} \\
\stackrel{0}{0}\end{array}$ & $\begin{array}{c}\sim \\
\stackrel{n}{y} \\
+1 \\
+ \\
+\end{array}$ & | & 1 & 1 & 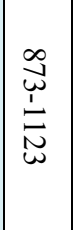 & $\overrightarrow{0}$ & 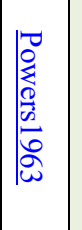 & $\begin{array}{l}\stackrel{N}{\vec{T}} \\
\stackrel{+}{+} \\
8\end{array}$ & | & | & 录 & 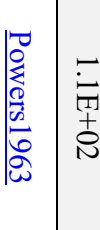 & 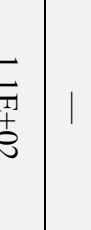 & $\mid$ & 1 & $\vec{u}$ & $\mid \begin{array}{l}0 \\
0 \\
0 \\
0 \\
0 \\
0 \\
0 \\
0 \\
0\end{array}$ \\
\hline 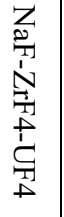 & \pm & $\begin{array}{l}0 \\
\dot{u} \\
\hat{i} \\
\dot{i} \\
\dot{0} \\
\dot{0} \\
\dot{0} \\
u\end{array}$ & 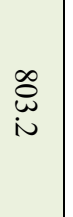 & 1 & 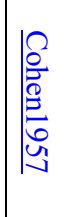 & 1 & | & | & \begin{tabular}{|l}
0 \\
i़ \\
T1 \\
8 \\
8
\end{tabular} & \begin{tabular}{|l}
$\dot{0}$ \\
$\dot{\tilde{T}}$ \\
$\dot{1}$ \\
$\dot{+}$
\end{tabular} & 1 & $u$ & 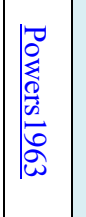 & $\begin{array}{l}\overrightarrow{\dot{\varphi}} \\
\dot{\varphi} \\
\dot{b}\end{array}$ & 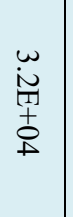 & | & 1 & 1 & 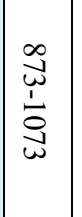 & $\overline{0}$ & 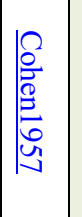 & | & | & | & | & 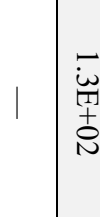 & 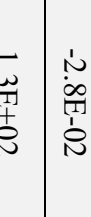 & | & 1 & $\vec{u}$ & 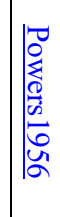 \\
\hline 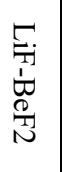 & t & $\begin{array}{l}0 \\
\dot{0} \\
0 \\
0 \\
0 \\
-\end{array}$ & $\underset{\substack{1 \\
\text { in }}}{ }$ & | & 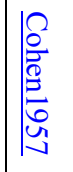 & 1 & | & | & 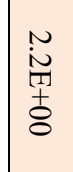 & 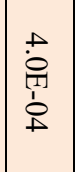 & 1 & $u$ & 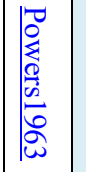 & $\begin{array}{l}\overline{\overrightarrow{\tilde{T}}} \\
\dot{\underline{v}} \\
\underline{n}\end{array}$ & $\begin{array}{l}0 \\
\dot{0} \\
\text { 故 }\end{array}$ & | & 1 & 1 & 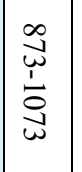 & $a$ & 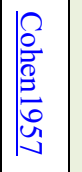 & | & | & | & | & | & \begin{tabular}{l|l}
1 & 1
\end{tabular} & | & 1 & 1 & $\|$ \\
\hline 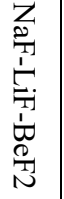 & 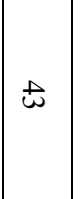 & 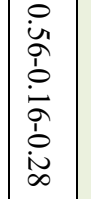 & $\stackrel{\vec{u}}{\vec{i}}$ & | & 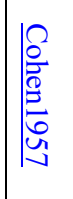 & 1 & | & | & 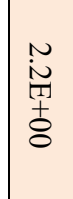 & 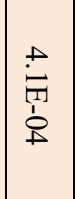 & 1 & $u$ & 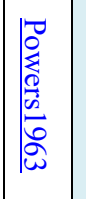 & $\begin{array}{l}\overrightarrow{\overline{\hat{T}}} \\
\dot{\underline{b}}\end{array}$ & 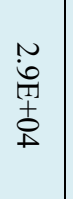 & & | & 1 & 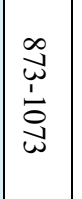 & $\overline{0}$ & 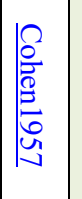 & I & I & I & | & | & \begin{tabular}{l|l}
1 & 1
\end{tabular} & | & 1 & 1 & $\|$ \\
\hline
\end{tabular}




\begin{tabular}{|c|c|c|c|c|c|c|c|c|c|c|c|c|c|c|c|c|c|c|c|c|c|c|c|c|c|c|c|c|c|c|c|}
\hline 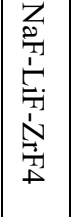 & \pm & 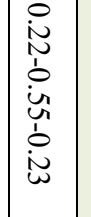 & 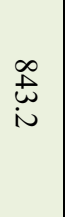 & I & 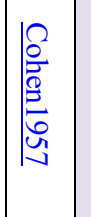 & | & | & | & 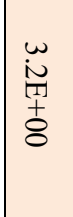 & $\begin{array}{l}\infty \\
\stackrel{\infty}{\bar{T}} \\
\dot{+} \\
\dot{\phi}\end{array}$ & 1 & $u$ & 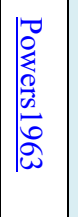 & 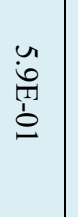 & \begin{tabular}{|l}
$w$ \\
0 \\
0 \\
01 \\
+1 \\
+
\end{tabular} & & | & $\mid$\begin{tabular}{l|l}
0 \\
\\
\cline { 1 - 1 }
\end{tabular} & অ & 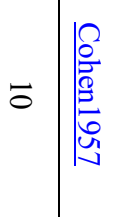 & I & 1 & 1 & | & 1 & 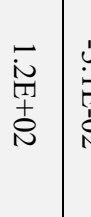 & 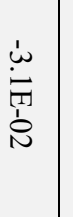 & | & | & $\bar{\sigma}$ & 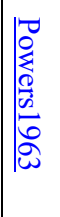 \\
\hline 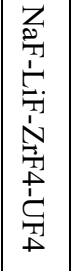 & to & 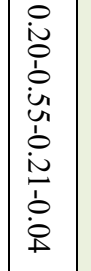 & $\underset{\substack{\infty \\
\infty}}{\infty}$ & I & 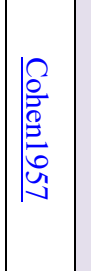 & | & 1 & 1 & 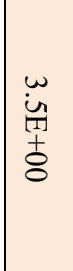 & 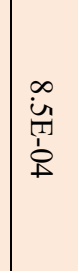 & 1 & un & 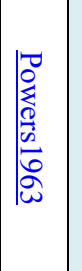 & 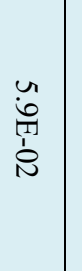 & \begin{tabular}{|l}
$w$ \\
0 \\
0 \\
01 \\
+1 \\
$c$
\end{tabular} & & | & 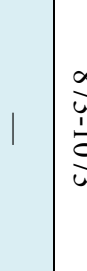 & 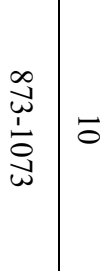 & 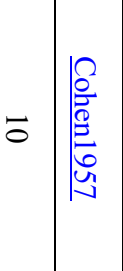 & | & I & 1 & | & 1 & 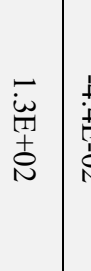 & 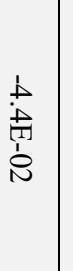 & | & | & $\vec{u}$ & 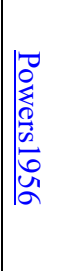 \\
\hline 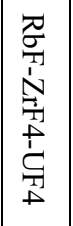 & t & $\begin{array}{l}0 \\
\dot{1} \\
\mathbf{\infty} \\
\dot{0} \\
\dot{+} \\
\dot{\infty} \\
\dot{0} \\
\dot{0}\end{array}$ & 尊 & 1 & 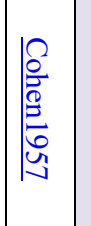 & | & 1 & 1 & 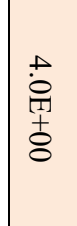 & \begin{tabular}{|l}
$\dot{0}$ \\
$\dot{\omega}$ \\
$\dot{\omega}$ \\
$\dot{1}$ \\
$\dot{+}$
\end{tabular} & 1 & un & 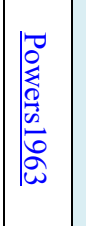 & $\begin{array}{l}\overrightarrow{\tilde{\tilde{N}}} \\
\dot{\omega} \\
\dot{v}\end{array}$ & $\begin{array}{l}\omega \\
\text { 离 } \\
+ \\
\dot{c}\end{array}$ & I & | & 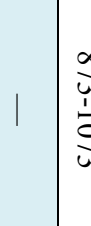 & 恿 & 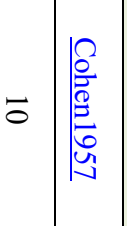 & $\mid$\begin{tabular}{|}
$\vec{y}$ \\
苟 \\
8 \\
\end{tabular} & I & 1 & 岕 & 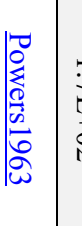 & 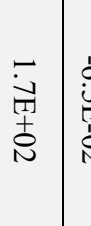 & 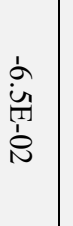 & 1 & | & $\sigma$ & 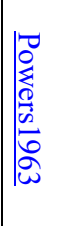 \\
\hline 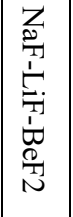 & $t$ & 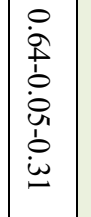 & 赵 & | & 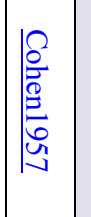 & I & | & 1 & $\begin{array}{l}\text { 走 } \\
\text { 孛 }\end{array}$ & \begin{tabular}{|l}
0 \\
$\dot{0}$ \\
$\dot{1}$ \\
$\dot{1}$
\end{tabular} & 1 & ur & 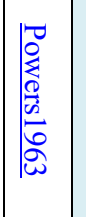 & 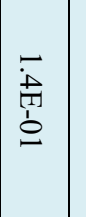 & 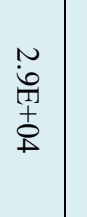 & | & I & $1 \mid$ & व & 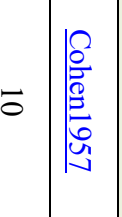 & 1 & 1 & | & I & | & 1 & I & 1 & | & | & 1 \\
\hline 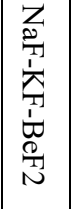 & $+\infty$ & $\begin{array}{l}0 \\
\dot{b} \\
0 \\
0 \\
\dot{\varphi} \\
\hat{b} \\
\dot{\omega} \\
o \\
o\end{array}$ & 赵 & | & 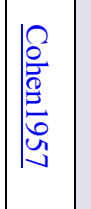 & I & $\mid$ & 1 & 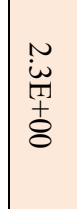 & \begin{tabular}{|l}
$\omega$ \\
0 \\
0 \\
1 \\
$\dot{p}$ \\
$\dot{p}$
\end{tabular} & 1 & $u$ & 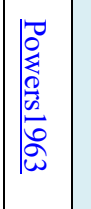 & $\begin{array}{l}\infty \\
\stackrel{\infty}{\mathbf{T}} \\
\stackrel{3}{\sim}\end{array}$ & 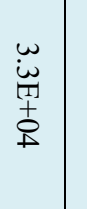 & & 1 & 1 & a & 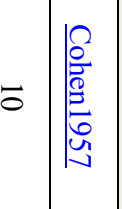 & 1 & | & | & I & | & 1 & I & | & I & | & 1 \\
\hline 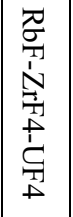 & b & 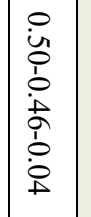 & $\underset{\sim}{\vec{~}}$ & | & 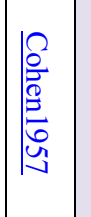 & 1 & | & $\mid$ & \begin{tabular}{|l}
+ \\
$\dot{0}$ \\
+1 \\
8 \\
8
\end{tabular} & $\mid \begin{array}{l}\dot{0} \\
\dot{w} \\
T \\
\dot{1} \\
\dot{f}\end{array}$ & 1 & $u$ & 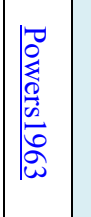 & $\begin{array}{l}\text { iे } \\
\text { 旁 }\end{array}$ & $\begin{array}{l}\text { 草 } \\
\text { 足 }\end{array}$ & & | & 1 & o & 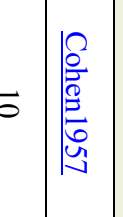 & 1 & I & | & I & | & 1 & I & | & | & | & \\
\hline
\end{tabular}




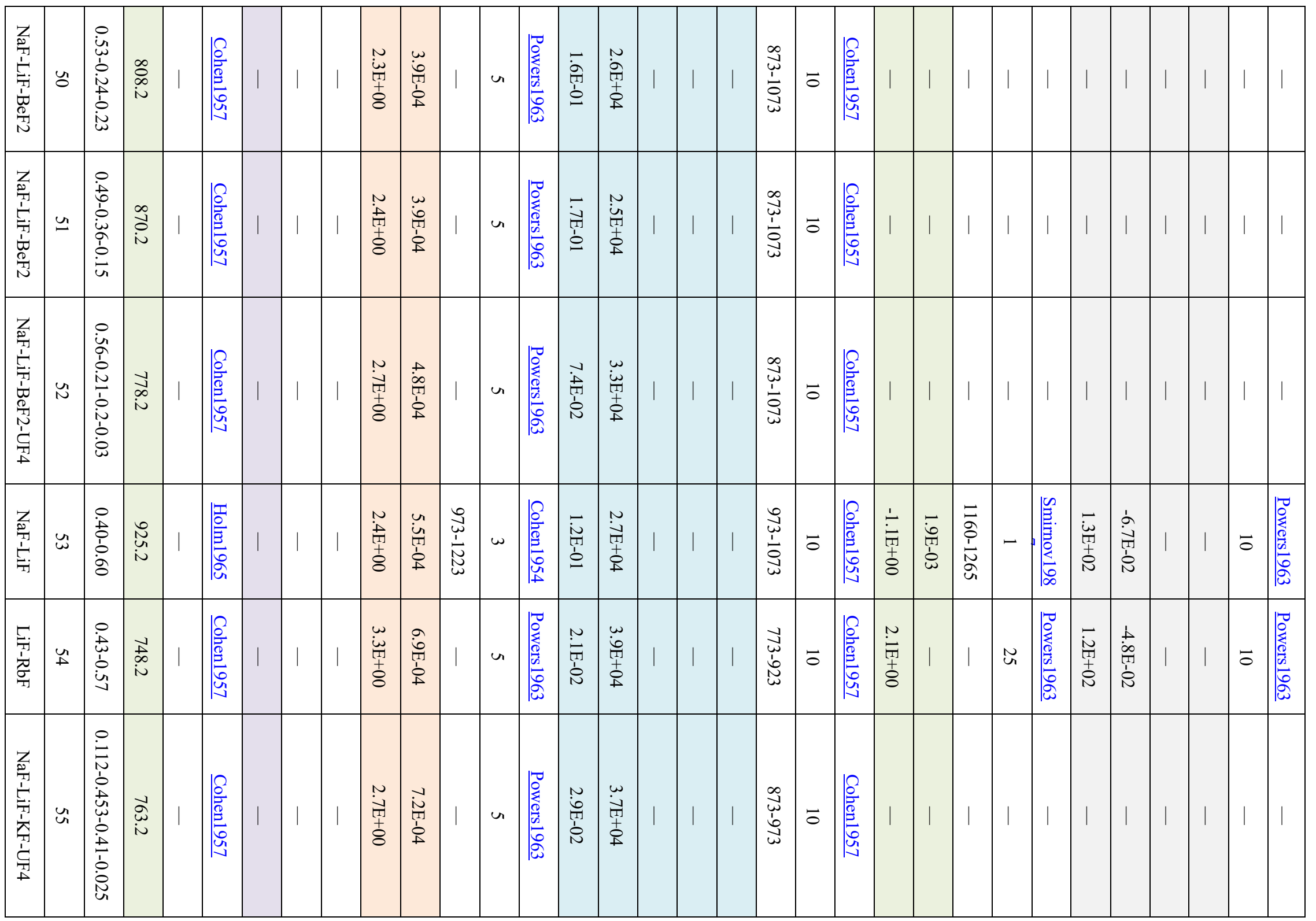




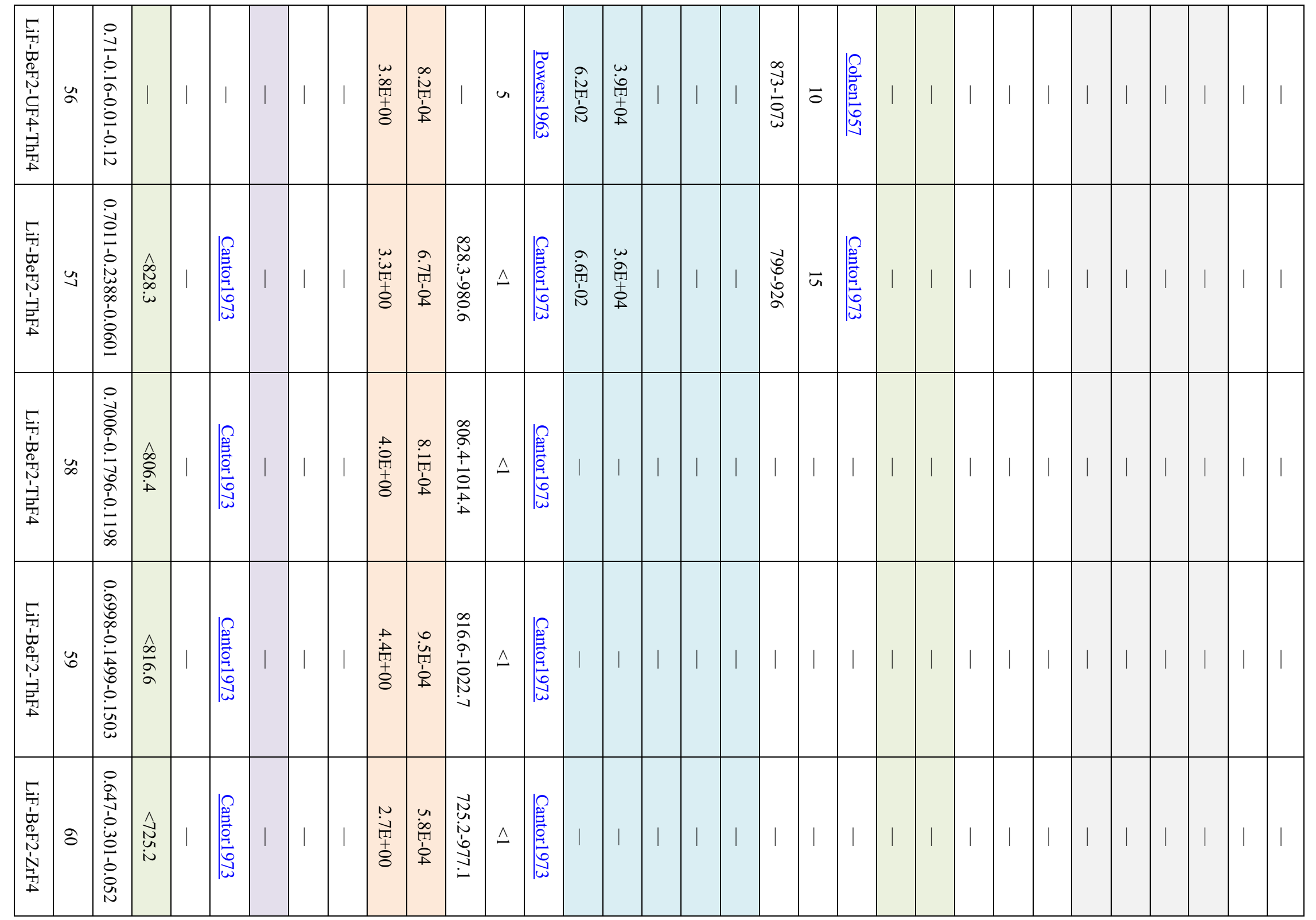




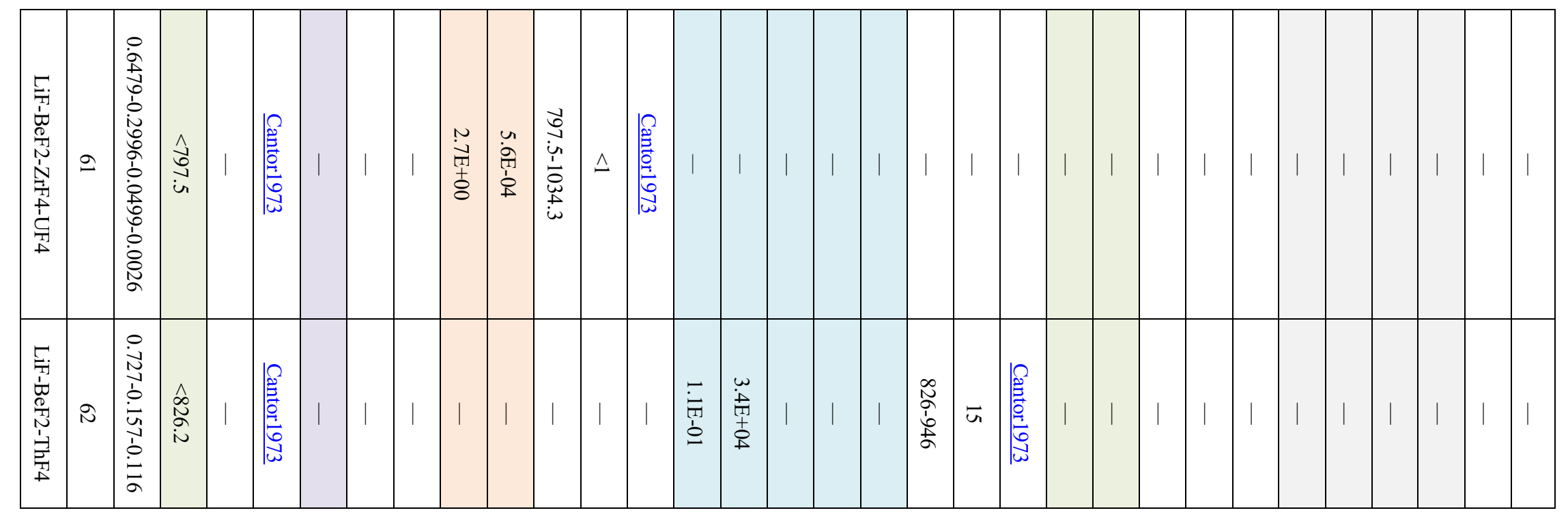

A-13 
APPENDIX B. MOLTEN SALT THERMOPHYISCAL DATA 



\section{APPENDIX B. MOLTEN SALT THERMOPHYISCAL DATA}

\begin{tabular}{|c|c|}
\hline References & Links \\
\hline $\begin{array}{l}\text { Abe, Y.; Kosugiyama, O.; Nagashima, A. Viscosity of LiF-BeF2 Eutectic Mixture } \\
\text { (XBeF2 =0.328) and LiF Single Salt at Elevated Temperatures. J. Nucl. Mater. 1981, } \\
99(2-3), 173-183 \text {. https://doi.org/10.1016/0022-3115(81)90186-0. }\end{array}$ & $\underline{\text { https://doi.org/10.1016/0022-3115(81)90186-0 }}$ \\
\hline $\begin{array}{l}\text { An, X.-H.; Cheng, J.-H.; Yin, H.-Q.; Xie, L.-D.; Zhang, P. Thermal Conductivity of } \\
\text { High Temperature Fluoride Molten Salt Determined by Laser Flash Technique. Int. J. } \\
\text { Heat Mass Transf. 2015, 90, 872-877. } \\
\text { https://doi.org/10.1016/j.ijheatmasstransfer.2015.07.042. }\end{array}$ & https://doi.org/10.1016/j.ijheatmasstransfer.2015.07.042 \\
\hline $\begin{array}{l}\text { Barin, I. Thermochemical Data of Pure Substances, Wiley, } 1995 . \\
\text { https://doi.org/10.1002/9783527619825. }\end{array}$ & https://doi.org/10.1002/9783527619825 \\
\hline $\begin{array}{l}\text { Barin, I.; Knacke, O.; Kubaschewski, O. Thermochemical Properties of Inorganic } \\
\text { Substances, Springer Berlin Heidelberg: Berlin, Heidelberg, } 1977 . \\
\text { https://doi.org/10.1007/978-3-662-02293-1. }\end{array}$ & https://doi.org/10.1007/978-3-662-02293-1 \\
\hline $\begin{array}{l}\text { Basin, A. S.; Kaplun, A. B.; Meshalkin, A. B.; Uvarov, N. F. The LiCl-KCl Binary } \\
\text { System. Russ. J. Inorg. Chem. 2008, } 53 \text { (9), 1509-1511. } \\
\text { https://doi.org/10.1134/S003602360809026X. }\end{array}$ & https://doi.org/10.1134/S003602360809026X \\
\hline $\begin{array}{l}\text { Beilmann, M.; Beneš, O.; Capelli, E.; Reuscher, V.; Konings, R. J. M.; Fanghänel, T. } \\
\text { Excess Heat Capacity in Liquid Binary Alkali-Fluoride Mixtures. Inorg. Chem. } \\
\text { 2013a, } 52 \text { (5), 2404-2411. https://doi.org/10.1021/ic302168g. }\end{array}$ & $\underline{\text { https://doi.org } / 10.1021 / \mathrm{ic} 302168 \mathrm{~g}}$ \\
\hline $\begin{array}{l}\text { Beilmann, M.; Beneš, O.; Konings, R. J. M.; Fanghänel, T. Thermodynamic } \\
\text { Assessment of the (LiF+UF3) and (NaF+UF3) Systems. J. Chem. Thermodyn. 2013b, } \\
\text { 57, 22-31. https://doi.org/10.1016/j.jct.2012.08.001. }\end{array}$ & $\underline{\text { https://doi.org/10.1016/j.jct.2012.08.001 }}$ \\
\hline
\end{tabular}


Bjorklund, C. W.; Reavis, J. G.; Leary, J. A.; Walsh, K. A. Phase Equilibria in the Binary Systems PuCl $2-\mathrm{NaCl}$ and $\mathrm{PuCl} 2$-LiCl. J. Phys. Chem. 1959, 63 (10),

1774-1777. https://doi.org/10.1021/j150580a049.

Borowitz, J. L.; Rafaeloff, R.; Roy, A. The Phase Diagrams of the Systems PbCl2UCl4 and PbCl2-UCl3. J. Electrochem. Soc. 1971, 118 (8), 1358.

https://doi.org/10.1149/1.2408322.

Brewer, L.; Somayajulu, G. R.; Brackett, E. Thermodynamic Properties of Gaseous Metal Dihalides. Chem. Rev. 1963, 63 (2), 111-121.

https://doi.org/10.1021/cr60222a002.

Brockner, W.; Tørklep, K.; Øye, H. A. Viscosity of Sodium Fluoride-Aluminium Fluoride Melt Mixtures. Berichte der Bunsengesellschaft für Phys. Chemie 1979, 83 (1), 12-19. https://doi.org/10.1002/bbpc.19790830103.

Cantor, S. Density and Viscosity of Several Molten Fluoride Mixtures. Oak Ridge National Laboratory, 1973. https://doi.org/10.2172/4419855.

https://doi.org/10.1021/j150580a049

(a)

https://doi.org/10.1149/1.2408322

https://doi.org/10.1021/cr60222a002

https://doi.org/10.1002/bbpc.19790830103

(1)

https://doi.org/10.2172/4419855

https://doi.org/10.1021/j100829a023

Cantor, S. Freezing point depressions in sodium fluoride. Effect of alkaline earth fluorides 1. J. Phys. Chem. 1961, 65 (12), 2208-2210.

https://doi.org/10.1021/j100829a023.

Cantor, S. Vapor Pressures of BeF 2 and NiF 2. J. Chem. Eng. Data 1965, 10 (3), 237-238. https://doi.org/10.1021/je60026a008.

$\underline{\text { https://doi.org/10.1021/je60026a008 }}$

Cantor, S.; Cooke, J. W.; Dworkin, A. S.; Robbins, G. D.; Thoma, R. E.; Watson, G. M. Physical properties of molten-salt reactor fuel, coolant, and flush salts. ORNLTM-2316. Oak Ridge National Laboratory, 1968. https://doi.org/10.2172/4492893.

Cantor, S.; Ward, W. T.; Moynihan, C. T. Viscosity and Density in Molten BeF 2 LiF Solutions. J. Chem. Phys. 1969, 50 (7), 2874-2879.

https://doi.org/10.1063/1.1671478.

https://doi.org/10.2172/4492893

https://doi.org/10.1063/1.1671478 
Chase, M. W.; Davies, C. A.; Downey, J. R.; Frurip, D. J.; Mcdonald, R. A.; Syverud, A. N. NIST-JANAF Thermodynamic Tables. J. Phys. Chem. Ref. Data Monogr. 1998, 9 .

Cho, K.; Irisawa, K.; Mochinaga, J.; Kuroda, T. Densities and Molar Volumes of Molten Rare-Earth Chlorides: $\mathrm{PrCl} 3, \mathrm{NdCl} 3, \mathrm{GdCl} 3$ and $\mathrm{DyCl} 3$. Electrochim. Acta 1972a, 17 (10), 1821-1827. https://doi.org/10.1016/0013-4686(72)85073-4.

Cho, K.; Kuroda, T. 希土類塩化物（ $\mathrm{PrCl} 3, \mathrm{NdCl} 3, \mathrm{GdCl} 3, \mathrm{DyCl} 3 ）$ 融解塩の粘度.

Denki Kagaku oyobi Kogyo Butsuri Kagaku 1972b, 40 (12), 878-881.

https://doi.org/10.5796/kogyobutsurikagaku.40.878.

Cibulková, J.; Chrenková, M.; Vasiljev, R.; Kremenetsky, V.; Boča, M. Density and Viscosity of the ( $\mathrm{LiF}+\mathrm{NaF}+\mathrm{KF})_{\text {eut }}(1)+\mathrm{K}_{2} \mathrm{TaF}_{7}(2)+\mathrm{Ta}_{2} \mathrm{O}_{5}$ (3) Melts. J. Chem. Eng. Data 2006, 51 (3), 984-987. https://doi.org/10.1021/je050490g.

Cohen, S. I.; Jones, T. N. A summary of density measurements on molten fluoride mixtures and a correlation for predicting densities of fluoride mixtures. ORNL-1702. Oak Ridge National Laboratory, 1954.

Cohen, S. I.; Jones, T. N. Viscosity measurements on molten fluoride mixtures. ORNL-2278. Oak Ridge National Laboratory, 1957. https://doi.org/10.2172/4803933.

D'Eye, R. W. M.; Martin, F. S. 349. The Barium Fluoride-Uranium Trifluoride System. J. Chem. Soc. 1957, 1847-1851. https://doi.org/10.1039/JR9570001847.

Dawson, R.; Brackett, E. B.; Brackett, T. E. A high temperature calorimeter; the enthalpies of $\alpha$-aluminum oxide and sodium chloride. J. Phys. Chem. 1963, 67 (8), 1669-1671. https://doi.org/10.1021/j100802a021.

Douglas, T. B.; Dever, J. L. Lithium Fluoride: Heat Content from 0 to $900^{\circ}$, the Melting Point and Heat of Fusion 1. J. Am. Chem. Soc. 1954, 76 (19), 4826-4829. https://doi.org/10.1021/ja01648a016. https://janaf.nist.gov/janaf4pdf.html

https://doi.org/10.1016/0013-4686(72)85073-4

https://doi.org/10.5796/kogyobutsurikagaku.40.878

https://doi.org/10.1021/je050490g

https://www.osti.gov/biblio/4334794-summary-densitymeasurements-molten-fluoride-mixtures-correlation-predictingdensities-fluoride-mixtures

https://doi.org/10.2172/4803933

https://doi.org/10.1039/JR9570001847

https://doi.org/10.1021/j100802a021

https://doi.org/10.1021/ja01648a016 
Douglas, T. B.; Victor, A. C. High-Temperature Heat Content. NBS Report 6297. US

Department of Commerce, National Bureau of Standards, 1959.

https://nvlpubs.nist.gov/nistpubs/Legacy/RPT/nbsreport6297.pdf

Druding, L. F.; Corbett, J. D. Lower Oxidation States of the Lanthanides.

Neodymium(II) Chloride and Iodide 1. J. Am. Chem. Soc. 1961, 83 (11), 2462-2467.

https://doi.org/10.1021/ja01472a010.

Dworkin, A. S.; Bredig, M. A. Enthalpy of Lanthanide Chlorides, Bromides, and Iodides from 298 to $1300 \mathrm{~K}$ : Enthalpies of Fusion and Transition. High Temp. Sci. 1971, 3, 1971.

Dworkin, A. S.; Bredig, M. A. Heat Content and Entropy of Strontium Chloride from $298^{\circ}$ to $1200^{\circ}$ K. J. Chem. Eng. Data 1963,8 (3), 416-417.

https://doi.org/10.1021/je60018a038.

Dworkin, A. S.; Bredig, M. A. The heats of fusion and transition of alkaline earth and rare earth metal halides. J. Phys. Chem. 1963a, 67 (3), 697-698.

https://doi.org/10.1021/j100797a038.

Efremova, R. I.; Matizen, E. V. Enthalpy of $\mathrm{BaF} 2, \mathrm{SrCl} 2$, and $\mathrm{SrF} 2$ at High

Temperatures. Izvest. Sib. Otd. Akad. Nauk S.S.S.R., Ser. Khim. Nauk 1970, 1, 3-12.

Ejima, T.; Shimakage, K.; Sato, Y.; Okuda, H.; Kumada, N.; Ishigaki, A. Viscosity Measurement of Alkali Chlorides with Capillary Viscometer. Nippon Kagaku Kaishi 1982, (6), 961-968. https://doi.org/10.1246/nikkashi.1982.961.

Gheribi, A. E.; Torres, J. A.; Chartrand, P. Recommended Values for the Thermal Conductivity of Molten Salts between the Melting and Boiling Points. Sol. Energy Mater. Sol. Cells 2014, 126, 11-25. https://doi.org/10.1016/j.solmat.2014.03.028.

https://www.osti.gov/biblio/4076058

https://doi.org/10.1021/je60018a038

https://doi.org/10.1021/j100797a038

Glushko, V. P. Thermicheskie Konstanty Veshchestv. 1994 
Glushko, V. P.; Medvedev, V. A.; Bergman, G. A.; Vasilev, B. P.; Gurvich, L. V; Alekseev, V. I.; Kolesov, V. P.; Yungman, V. S.; Ioffe, N. T.; Vorabev, A. F.

Thermicheskie Konstanty Veshchestv. Russia, 1972.

$\underline{\text { http://chem.msu.ru/cgi-bin/tkv.pl }}$

Haendler, H. M.; Sennett, P. S.; Wheeler, C. M. The System LiF-LiCl, LiF-NaCl, LiF-KCl. J. Electrochem. Soc. 1959, 106 (3), 264. https://doi.org/10.1149/1.2427319.

https://doi.org/10.1149/1.2427319

Hayashi, H.; Kato, Y.; Ogawa, T.; Sato, Y. Development of Viscometers for Molten Salts. Japan Atomic Energy Research Institute,

Japan, 1997.

Hill, D. G.; Cantor, S.; Ward, W. T. Molar Volumes in the LiFThF4 System. J. Inorg. Nucl. Chem. 1967, 29 (1), 241-243. https://doi.org/10.1016/0022-1902(67)80163-5.

https://doi.org/10.1016/0022-1902(67)80163-5

Holm, J. L.; Pikas, A.-L.; Ryhage, R.; Nielsen, P. H.; Sjöberg, B.; Larsen, E. Phase Relations in the Systems NaF-LiF, NaF-KF, and NaF-RbF. Acta Chem. Scand. 1965, 19 (3), 638-644. https://doi.org/10.3891/acta.chem.scand.19-0638.

Janz, G. J. Molten Salts Handbook, Elsevier: New York, 1967.

https://doi.org/10.1016/B978-0-123-95642-2.X5001-1.

https://inis.iaea.org/search/search.aspx?orig q=RN:28057468

Janz, G. J. Thermodynamic and Transport Properties for Molten Salts: Correlation

Equations for Critically Evaluated Density, Surface Tension, Electrical Conductance, and Viscosity Data. J. Phys. Chem. Ref. Data 1988, 17.

Janz, G. J.; Kelly, F. J.; Pérano, J. L. Melting and Pre-Melting Effects in the Alkaline Earth Halides. Trans. Faraday Soc. 1963, 59 (0), 2718-2722.

https://doi.org/10.1039/TF9635902718.

Jerden, J. Molten Salt Thermophysical Properties Database Development: 2019 Update. ANL/CFCT-19/6. Argonne National Laboratory, 2019.

https://doi.org/10.2172/1559846.

https://doi.org/10.3891/acta.chem.scand.19-0638

https://doi.org/10.1016/B978-0-123-95642-2.X5001-1

https://srd.nist.gov/JPCRD/jpcrdS2Vol17.pdf

https://doi.org/10.1039/TF9635902718

https://doi.org/10.2172/1559846 
Johnson, J. W.; Bredig, M. A. Miscibility of Metals with Salts in the Molten State. III. The Potassium-Potassium Halide Systems. J. Phys. Chem. 1958, 62 (5), 604-607. https://doi.org/10.1021/j150563a021.

Katyshev, S. F.; Desyatnik, V. N. Density and Surface Tension of Uranium

Tetrachloride Melts with Magnesium and Calcium Chlorides. Sov. At. Energy 1981, 51 (6), 810-812. https://doi.org/10.1007/BF01121688.

Keneshea, R. J.; Cubicciotti, D. Lanthanum-Lanthanum Trichloride Phase Diagram. J. Chem. Eng. Data 1961, 6 (4), 507-509. https://doi.org/10.1021/je60011a008.

https://doi.org/10.1021/j150563a021

\author{
(a)
}

https://doi.org/10.1007/BF01121688

(1)

https://doi.org/10.1021/je60011a008

Kirshenbaum, A. D.; Cahill, J. A. The Density of Molten Thorium and Uranium Tetrafluorides. J. Inorg. Nucl. Chem. 1961, 19 (1-2), 65-68.

https://doi.org/10.1016/0022-1902(61)80047-X.

Kirshenbaum, A. D.; Cahill, J. A.; Stokes, C. S. The Density of Molten Metal

Fluorides in the Range of $1600^{\circ}-2500^{\circ}$ K. J. Inorg. Nucl. Chem. 1960, 15 (3-4), $297-$

304. https://doi.org/10.1016/0022-1902(60)80057-7.

Klemm, W. Dichtemessungen an Geschmolzenen Chloriden. Zeitschrift fur Anorg. und Allg. Chemie 1926, 152 (1), 235-251. https://doi.org/10.1002/zaac.19261520127.

https://doi.org/10.1016/0022-1902(61)80047-X

https://doi.org/10.1016/0022-1902(60)80057-7

https://doi.org/10.1002/zaac.19261520127

Knacke, O.; Kubaschewski, O.; Hesselmann, K. Thermochemical Properties of Inorganic Substances, 2nd ed. Barin, I., Ed., Elsevier Science \& Technology, 1991.

https://books.google.com/books?id=5T9RAAAAMAAJ

Kojima, H.; Whiteway, S. G.; Masson, C. R. Melting Points of Inorganic Fluorides. Can. J. Chem. 1968, 46 (18), 2968-2971. https://doi.org/10.1139/v68-494.

https://doi.org/10.1139/v68-494

Kulifeev, V. K.; Panchishnyi, V. I.; Stanolevich, G. P. Density, viscosity, and surface tension of uranium tetrafluoride. 1971. 
Landolt. Pure Substances. Part 3 _Compounds from CoCl3_g to Ge3N4; LandoltBörnstein - Group IV Physical Chemistry; Springer-Verlag: Berlin/Heidelberg, 2000, vol. 19 A3. https://doi.org/10.1007/b60167.

Langer, S.; Blankenship, F. F. The Vapour Pressure of Uranium Tetrafluoride. J. Inorg. Nucl. Chem. 1960, 14 (1-2), 26-31. https://doi.org/10.1016/00221902(60)80194-7.

Lemire, R. J.; Fuger, J.; Nitsche, H.; Potter, P.; Rand, M. H.; Rydberg, J.; Spahiu, K.; Sullivan, J. C.; Ullmann, W. J.; Vitorge, P.; Wanner, H. Chemical Thermodynamics of Neptunium and Plutonium; Elsevier: Amsterdam, 2001, vol. 4.

https://doi.org/10.1007/b60167

(1)

https://doi.org/10.1016/0022-1902(60)80194-7

https://www.oecd-nea.org/jems/pl_13530

Manohar S. Sohal; Matthias A. Ebner; Piyush Sabharwall; Phil Sharpe. Engineering Database of Liquid Salt Thermophysical and Thermochemical Properties. INL/EXT10-18297. Idaho National Laboratory, 2010. https://doi.org/10.2172/980801.

McDonald, R. A.; Oetting, F. L. The Thermodynamic Properties and Allotropy of Beryllium Chloride between 13 and $715^{\circ}$ K. 1. J. Phys. Chem. 1965, 69 (11), 38393845. https://doi.org/10.1021/j100895a033.

Mochinaga, J.; Cho, K.; Takagi, R.; Kuroda, T. UCl3浴およびUCl3-KCl系混合浴 の密度と当量導電率. Denki Kagaku oyobi Kogyo Butsuri Kagaku 1969, 37 (9), 654-658. https://doi.org/10.5796/kogyobutsurikagaku.37.654.

Moore, G. E. Heat Contents at High Temperatures of the Anhydrous Chlorides of Calcium, Iron, Magnesium and Manganese 1. J. Am. Chem. Soc. 1943, 65 (9), 17001703. https://doi.org/10.1021/ja01249a014.

Moynihan, C. T.; Cantor, S. Viscosity and Its Temperature Dependence in Molten BeF 2. J. Chem. Phys. 1968, 48 (1), 115-119. https://doi.org/10.1063/1.1664455.

MUKERJI, J. Phase Equilibrium Diagram CaO-CaF2-2CaO.SiO2. J. Am. Ceram. Soc. 1965, 48 (4), 210-213. https://doi.org/10.1111/j.1151-2916.1965.tb14715.x.

https://doi.org/10.2172/980801

https://doi.org/10.1021/j100895a033

https://doi.org/10.5796/kogyobutsurikagaku.37.654

hes

https://doi.org/10.1021/ja01249a014

$\underline{\text { https://doi.org/10.1063/1.1664455 }}$

https://doi.org/10.1111/j.1151-2916.1965.tb14715.x 
Murgulescu, I. G.; Telea, C. Heat capacities in molten alkaline halides. Rev. Roum.

https://pascal-

Chim. 1977, 22 (5), 683-689.

francis.inist.fr/vibad/index.php?action=getRecordDetail\&idt=PAS

CAL7860022220

Nagasaka, Y.; Nakazawa, N.; Nagashima, A. Experimental Determination of the Thermal Diffusivity of Molten Alkali Halides by the Forced Rayleigh Scattering Method. I. Molten $\mathrm{LiCl}, \mathrm{NaCl}, \mathrm{KCl}, \mathrm{RbCl}$, and CsCl. Int. J. Thermophys. 1992, 13 (4), 555-574. https://doi.org/10.1007/BF00501941.

Naylor, B. F. Heat Contents at High Temperatures of Magnesium and Calcium Fluorides 1. J. Am. Chem. Soc. 1945, 67 (1), 150-152.

https://doi.org/10.1021/ja01217a052.

O'Brien, C. J.; Kelley, K. K. High Temperature Heat Contents of Cryolite,

Anhydrous Aluminum Fluoride and Sodium Fluoride. J. Am. Chem. Soc. 1957, 79

(21), 5616-5618. https://doi.org/10.1021/ja01578a009.

Paucirova, M.; Matiasovsky, K.; Malinovsky, M. Volume properties of melts of systems lif-aif3 and naf-aif3. Rev. Roum. Chim. 1970, 15 (1), 33-41.

Powers, W. D.; Blalock, G. C. Enthalpies and heat capacities of solid and molten fluoride mixtures. Oak Ridge National Laboratory, 1956.

https://doi.org/10.2172/4794927.

Powers, W. D.; Cohen, S. I.; Greene, N. D. Physical Properties of Molten Reactor Fuels and Coolants. Nucl. Sci. Eng. 1963, 17 (2), 200-211.

https://doi.org/10.13182/NSE63-5.

Rand, M. H., Fuger, J., The thermodynamic properties of the transuranium halides: Part 1, Neptunium and plutonium halides, European Commission Joint Research Centre report 17332 EN, ITU, Karlsruhe, 2000.

Redkin, A.; Korzun, I.; Yaroslavtseva, T.; Reznitskikh, O.; Zaikov, Y. Isobaric Heat Capacity of Molten Halide Eutectics. J. Therm. Anal. Calorim. 2017, 128 (1), 621626. https://doi.org/10.1007/s10973-016-5869-9.

https://doi.org/10.1007/BF00501941

https://doi.org/10.1021/ja01217a052

https://doi.org/10.1021/ja01578a009

https://doi.org/10.2172/4794927

https://doi.org/10.13182/NSE63-5

https://doi.org/10.1007/s10973-016-5869-9 
Rogers, D. J.; Yoko, T.; Janz, G. J. Fusion Properties and Heat Capacities of the Eutectic Lithium Fluoride-Sodium Fluoride-Potassium Fluoride Melt. J. Chem. Eng. Data 1982, 27 (3), 366-367. https://doi.org/10.1021/je00029a041.

https://doi.org/10.1021/je00029a041

Rosenthal, M. W.; Briggs, R. B.; Kasten, P. R. molten-salt reactor program semiannual progress report for period ending August 31, 1968. ORNL-4344. Oak Ridge National Laboratory, 1969. https://doi.org/10.2172/4805939.

Ruff, O. Fortschritte in Der Chemie Des Fluors. Zeitschrift für Angew. Chemie 1928, 41 (27), 737-740. https://doi.org/10.1002/ange.19280412703.

Ruff, O.; Mugdan, S. Arbeiten Aus Dem Gebiet Hoher Temperaturen XIII. Die

Messung von Dampfdrucken Bei Hohen Temperaturen Und Die Dampfdrucke Der

Alkalihalogenide. Zeitschrift fur Anorg. und Allg. Chemie 1921, 117 (1), 147-171. https://doi.org/10.1002/zaac.19211170111.

Ruff, O.; Schmidt, G.; Mugdan, S. Arbeiten Aus Dem Gebiet Hoher Temperaturen. XV. Die Dampfdrucke Der Alkalifluoride. Zeitschrift fur Anorg. und Allg. Chemie 1922, 123 (1), 83-88. https://doi.org/10.1002/zaac.19221230108.

Shishido, H.; Yusa, N.; Hashizume, H.; Ishii, Y.; Ohtori, N. Thermal Design Investigation for a Flinabe Blanket System. Fusion Sci. Technol. 2017, 72 (3), 1-7. https://doi.org/10.1080/15361055.2017.1330623.

Smirnov, M. V.; Khokhlov, V. A.; Filatov, E. S. Thermal Conductivity of Molten Alkali Halides and Their Mixtures. Electrochim. Acta 1987, 32 (7), 1019-1026. https://doi.org/10.1016/0013-4686(87)90027-2.

Smirnov, M. V.; Khoklov, V. A.; Stepanov, V. P. Viscosity of Fused Lanthanum Trichloride and Its Mixture With Dichloride. Russ. J. Phys. Chem. USSR 1966, 40 (6), 671 .

Spedding, F. H.; Beaudry, B. J.; Henderson, D. C.; Moorman, J. High Temperature Enthalpies and Related Thermodynamic Functions of the Trifluorides of Sc, Ce, Sm, Eu,Gd, Tb, Dy, Er, Tm, and Yb. J. Chem. Phys. 1974, 60 (4), 1578-1588.

https://doi.org/10.1063/1.1681233.

https://doi.org/10.2172/4805939

https://doi.org/10.1002/ange.19280412703

https://doi.org/10.1002/zaac.19211170111

https://doi.org/10.1002/zaac.19221230108

https://doi.org/10.1080/15361055.2017.1330623

(n)

https://doi.org/10.1016/0013-4686(87)90027-2

https://doi.org/10.1063/1.1681233 
Spedding, F. H.; Henderson, D. C. High-Temperature Heat Contents and Related Thermodynamic Functions of Seven Trifluorides of the Rare Earths: Y, La, Pr, Nd, Gd, Ho, and Lu. J. Chem. Phys. 1971, 54 (6), 2476-2483.

https://doi.org/10.1063/1.1675202

https://doi.org/10.1063/1.1675202.

Takeda, O.; Hoshino, Y.; Anbo, Y.; Yanagase, K.; Aono, M.; Sato, Y. Viscosity of Molten Alkaline-Earth Fluorides. Int. J. Thermophys. 2015, 36 (4), 648-657. https://doi.org/10.1007/s10765-014-1828-5.

Thoma, R. E.; Insley, H.; Friedman, H. A.; Hebert, G. M. Equilibrium Phase Diagram of the Lithium Fluoride-Beryllium Fluoridezirconium Fluoride System. J. Nucl. Mater. 1968, 27 (2), 166-180. https://doi.org/10.1016/0022-3115(68)90120-7.

Thoma, R. E.; Insley, H.; Friedman, H. A.; Weaver, C. F. PHASE EQUILIBRIA IN THE SYSTEMS BeF 2 -ThF 4 AND LiF-BeF 2 -ThF 4. J. Phys. Chem. 1960, 64 (7), 865-870. https://doi.org/10.1021/j100836a010.

Toerklep, K.; Oeye, H. A. Viscosity of Molten Alkaline-Earth Chlorides. J. Chem. Eng. Data 1982, 27 (4), 387-391. https://doi.org/10.1021/je00030a006.

Toerklep, K.; Oeye, H. A. Viscosity of the Eutectic Lithium Fluoride-Sodium Fluoride-Potassium Fluoride Melt (FLINAK). J. Chem. Eng. Data 1980, 25 (1), 1617. https://doi.org/10.1021/je60084a007.

Torklep, K.; Oye, H. A. An Absolute Oscillating-Cylinder (or Cup) Viscometer for High Temperatures. J. Phys. E. 1979, 12 (9), 875-885. https://doi.org/10.1088/0022$3735 / 12 / 9 / 021$.

Van Artsdalen, E. R.; Yaffe, I. S. Electrical Conductance and Density of Molten Salt Systems: KCl-LiCl, KCl-NaCl and KCl-KI. J. Phys. Chem. 1955, 59 (2), 118-127. https://doi.org/10.1021/j150524a007.

Von Wartenberg, H.; Schulz, H. Der Dampfdruck Einiger Salze. II. Zeitschrift für Elektrochemie und Angew. Phys. Chemie 1921, 27 (23-24), 568-573. https://doi.org/10.1002/bbpc.19210272306.

https://doi.org/10.1021/je00030a006

https://doi.org/10.1007/s10765-014-1828-5

https://doi.org/10.1016/0022-3115(68)90120-7

https://doi.org/10.1021/j100836a010

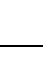

https://doi.org/10.1021/je60084a007

$\underline{\text { https://doi.org/10.1088/0022-3735/12/9/021 }}$

https://doi.org/10.1021/j150524a007

https://doi.org/10.1002/bbpc.19210272306 
Wakao, M.; Minami, K.; Nagashima, A. Viscosity Measurements of Molten LiCl in the Temperature Range 886-1275 K. Int. J. Thermophys. 1991, 12 (2), 223-230.

https://doi.org/10.1007/BF00500748

https://doi.org/10.1007/BF00500748

Westrum, E. F.; Wallmann, J. C. The Melting Point and the Heat of Sublimation of Plutonium Trifluoride 1. J. Am. Chem. Soc. 1951, 73 (7), 3530-3531.

https://doi.org/10.1021/ja01151a535.

Williams, D. F. Assessment of Candidate Molten Salt Coolants for the Advanced High Temperature Reactor (AHTR). Oak Ridge National Laboratory, 2006a.

https://doi.org/10.2172/885975.

Williams, D. F. Assessment of Candidate Molten Salt Coolants for the NGNP/NHI Heat-Transfer Loop. Oak Ridge National Laboratory, $2006 \mathrm{~b}$. https://doi.org/10.2172/1360677.

Xu, X.; Wang, X.; Li, P.; Li, Y.; Hao, Q.; Xiao, B.; Elsentriecy, H.; Gervasio, D. Experimental Test of Properties of $\mathrm{KCl}-\mathrm{MgCl} 2$ Eutectic Molten Salt for Heat

Transfer and Thermal Storage Fluid in Concentrated Solar Power Systems. J. Sol. Energy Eng. 2018, 140 (5). https://doi.org/10.1115/1.4040065.

Yaffe, I. S.; Van Artsdalen, E. R. Chemistry Division Semiannual Report, ORNL2159. Oak Ridge National Laboratory, 1956.

https://doi.org/10.1021/ja01151a535

https://doi.org/10.2172/885975

https://doi.org/10.2172/1360677

https://doi.org/10.1115/1.4040065 


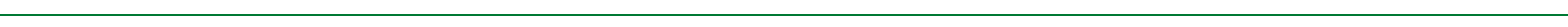

